\title{
Un petit ensemble funéraire à crémation du Haut- Empire au « Marais » à Gorges (Loire-Atlantique)
}

A small funeral group of cremation from the Early Roman Empire in "Le Marais" at Gorges (Loire-Atlantique)

Eine kleine Gruppe römischer Brandgräber der frühen römischen Kaiserzeit bei Gorges „Le Marais“ (Loire-Atlantique, Frankreich) Un pequeño conjunto funerario a cremación del Alto Imperio en el "Marais" en Gorges (Loire-Atlantique)

\section{Patrick Bellanger, Joël Cornec, Isabelle Le Goff et Jérôme Pascal}

\section{OpenEdition}

\section{Journals}

Édition électronique

URL : https://journals.openedition.org/rao/3476

DOI : $10.4000 /$ rao.3476

ISSN : 1775-3732

Éditeur

Presses universitaires de Rennes

Édition imprimée

Date de publication : 31 décembre 2016

Pagination : 221-242

ISBN : 978-2-7535-5683-6

ISSN : 0767-709X

Référence électronique

Patrick Bellanger, Joël Cornec, Isabelle Le Goff et Jérôme Pascal, « Un petit ensemble funéraire à crémation du Haut-Empire au « Marais » à Gorges (Loire-Atlantique) », Revue archéologique de l'Ouest [En ligne], 33 | 2016, mis en ligne le 31 juillet 2019, consulté le 22 août 2022. URL : http:// journals.openedition.org/rao/3476 ; DOl : https://doi.org/10.4000/rao.3476 


\title{
Un petit ensemble funéraire à crémation du Haut-Empire au " Marais » à Gorges (Loire-Atlantique)
}

\author{
A Small Funeral Group of Cremation from the Early Roman Empire \\ in "Le Marais" at Gorges (Loire-Atlantique)
}

\section{Patrick Bellanger ${ }^{a}$, Joël Cornec ${ }^{b}$, Isabelle Le GofF ${ }^{c}$ et Jérôme Pascal ${ }^{\mathrm{d}}$}

\begin{abstract}
Résumé : À proximité d'un habitat rural d'époque romaine, deux sépultures à crémation en dépôt secondaire datées du milieu du II ${ }^{\mathrm{e}}$ siècle après J.-C. ont été découvertes au lieu-dit «Le Marais » à Gorges. L’une d'elles renfermait un dépôt de crémation mixte, associée à une troisième fosse contenant des résidus de la même crémation. Cependant, il n'a pas été possible d'établir clairement si les deux premières crémations, contenant les restes d'un jeune enfant, étaient des sépultures distinctes ou si les restes de la même personne avaient été répartis entre les deux dépôts.
\end{abstract}

\begin{abstract}
Two secondary cremation burials dating from the middle of the $2^{\text {nd }}$ century AD were discovered in "Le Marais" at Gorges, near a roman rural settlement. One of the burials contained a mixed cremation deposit, the residues of this same cremation having been deposited in a third pit. However, it was not possible to clearly establish if the first two cremations, both containing the remains of a young child, were separate burials or if the remains of the same individual had been divided up between the two deposits.
\end{abstract}

Mots clés : Loire-Atlantique, Haut-Empire, dépôt secondaire de crémation, dépôt de résidus, fosse cendreuse.

Keywords: Loire-Atlantique, Gorges, Early Roman Empire, secondary cremation deposit, cremation residues, ashy pit.

\section{Contexte de la déCouverte}

La commune de Gorges (Loire-Atlantique) se situe dans la partie septentrionale de la cité des Pictons, à une vingtaine de kilomètres au sud-est de Nantes, sur la rive gauche de la Sèvre nantaise, affluent de la Loire s'écoulant ici dans un vallon particulièrement encaissé. Le lieu de la découverte est localisé au sud du bourg de Gorges, près de la limite entre cette commune et celle de Clisson, à environ huit cents mètres de la Sèvre nantaise et dans une légère cuvette de plateau occupée par une prairie marécageuse établie à 38 m NGF (fig. 1). Le socle rocheux du secteur appartient au massif intrusif granitique de Clisson-Mortagne et se présente sous une forme plus ou moins altérée. Le substrat se

\footnotetext{
a Patrick BELLANGER, chargé d'opérations et de recherches, Inrap Grand-Ouest, Centre de recherches archéologiques, 4 rue du Tertre 44470 CARQUEFOU. (patrick.bellanger@inrap.fr)

${ }^{b}$ Joël CORNEC, assistant d'études et d'opérations, Inrap Grand-Ouest, Centre de recherches archéologiques, 4 rue du Tertre 44470 CARQUEFOU. (joel. cornec@inrap.fr)

'Isabelle LE GOFF, ingénieur d'études en archéologie funéraire, Inrap Grand-Est-Nord, Centre de recherches archéologiques, 28 rue Robert-Fulton, 51689 REIMS Cedex 2. (isabelle.le-goff@inrap.fr)

'Jérôme PASCAL, chargé d'opération et de recherche, Inrap Grand-Ouest, Centre de recherches archéologiques, 31 allée des Justices, 85200 FONTENAY-LECOMTE. (jerome.pascal@inrap.fr)
} 


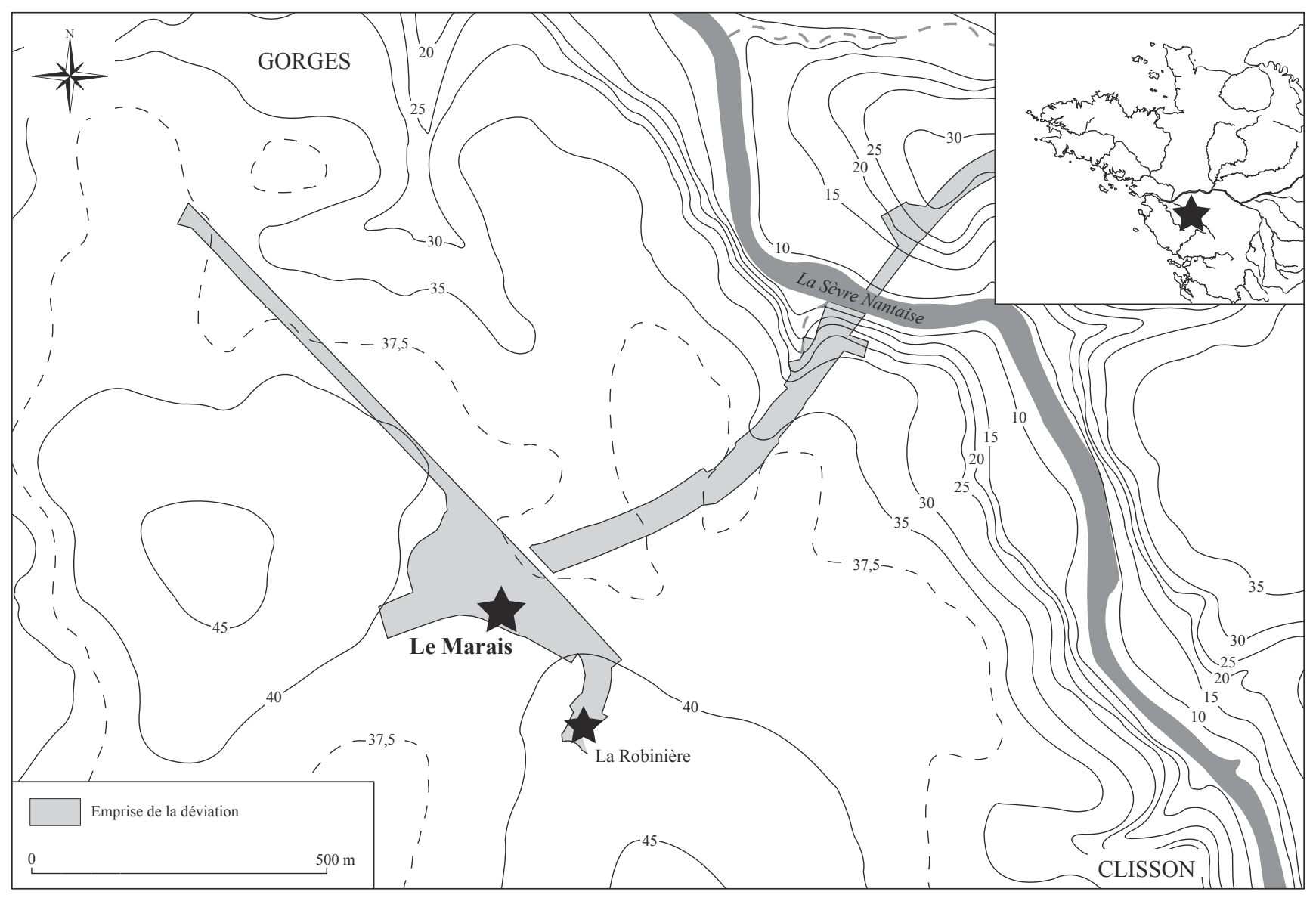

Figure 1 : Gorges, «Le Marais » : localisation géographique. DAO : P. Bellanger.

Figure 1: Geographical location map.

compose de formations sablo-argileuses (formations plioquaternaires) surmontées par des limons éoliens de couleur brun clair à orangé.

En 2002, le Service régional de l'Archéologie des Pays de la Loire a prescrit un diagnostic archéologique sur l'emprise du contournement routier au nord et à l'est de la ville de Clisson (Bellanger, 2002). La prospection mécanique du secteur, relativement étendu à cet endroit du projet de déviation, a été effectuée en tranchées implantées en quinconce, qui n’ont, pour la majorité, livré que des fossés de parcellaires de datation indéterminée. Outre un indice d'occupation protohistorique au Chaintreau à Clisson et une portion d'enclos d'époque romaine au Barillet à Gétigné, l'intervention a également permis de mettre au jour trois fosses en lien avec les pratiques de la crémation dont la composition évoque différentes phases des funérailles. Datant du HautEmpire, ces fosses étaient localisées à l'extrémité nord-est du tracé routier, au lieu-dit Le Marais, à environ deux cents mètres au nord-ouest d'un indice d'occupation antique détecté lors du même diagnostic à La Robinière (fig. 2). Le caractère particulièrement humide du terrain, peu propice au labour, est sans doute à l'origine de la bonne conservation de cet ensemble funéraire.

Une tranchée réalisée à proximité de la limite sud de l'emprise a toutefois révélé la présence d'une fosse comblée par des résidus de combustion du bûcher mêlés de petits fragments de verre fondus et de céramiques brûlées (F1). L'extension de ce sondage a finalement permis de détecter deux autres fosses disposées au nord-est de la première, qui correspondent à des sépultures avec une urne cinéraire (F2 et F3). Après un nettoyage de surface suivi d'un relevé en plan, les trois fosses ont fait l'objet d'une fouille par moitié afin d'observer la coupe stratigraphique de leur comblement, puis d'une fouille intégrale par unités stratigraphiques. Les urnes mises au jour, le mobilier qui les accompagnait ainsi que les sédiments charbonneux et cendreux ont été prélevés en prévision d'études et analyses ultérieures. La fouille minutieuse des urnes, le tamisage des prélèvements et les analyses anthracologiques et carpologiques des charbons de bois ont été effectués en 2006 grâce au cofinancement du Service 
Figure 2 : Gorges, «Le Marais ": localisation et plan des vestiges gallo-romains. DAO : J. Cornec.

Figure 2: Location and plan of the gallo-roman remnants.

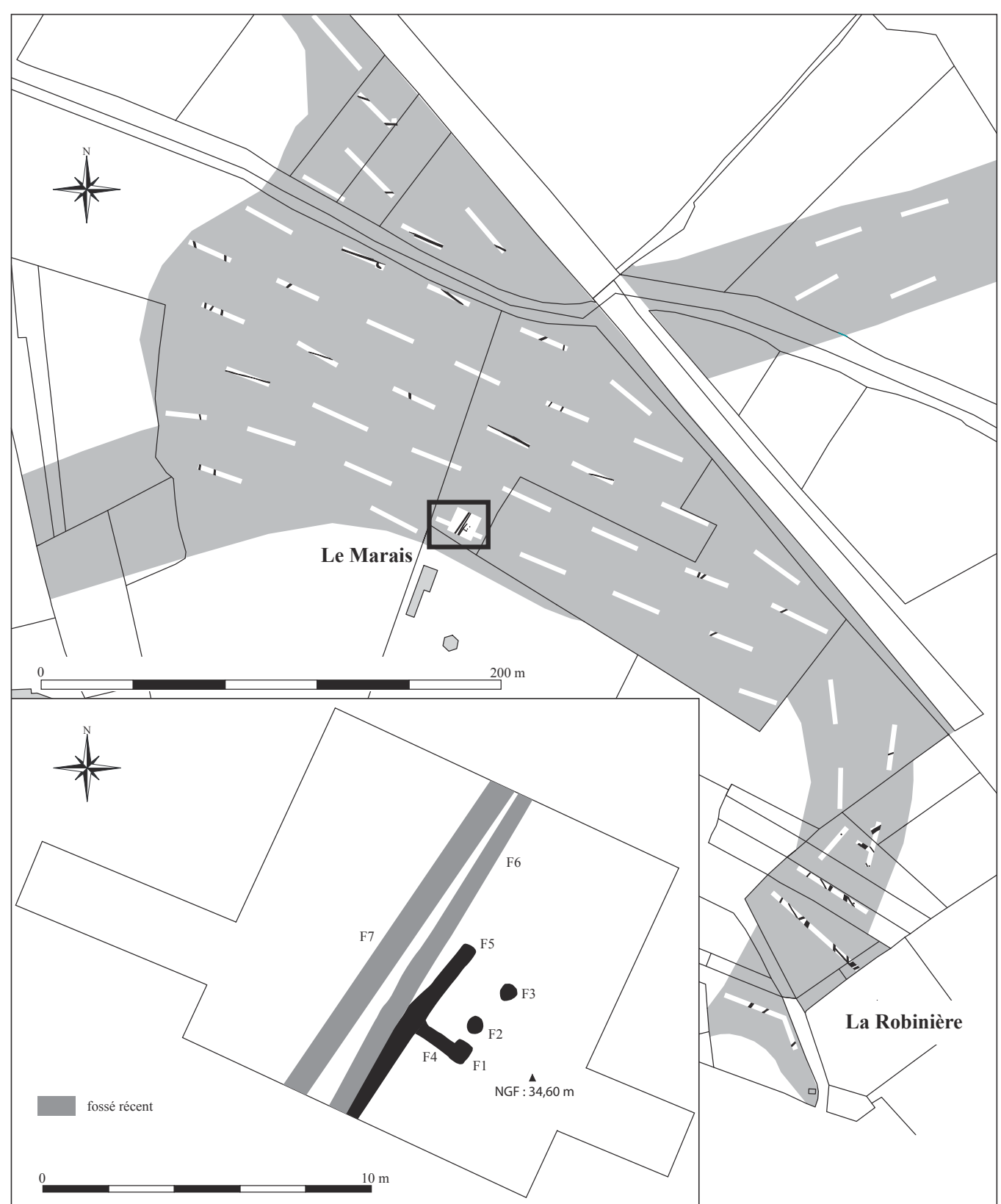

régional de l'Archéologie des Pays de la Loire et de l'Institut national de recherches archéologiques préventives (Inrap).

\section{LES TROIS DÉPÔTS FUNÉRAIRES}

L'ensemble funéraire du Marais se compose de trois fosses - F1, F2 et F3 - espacées de 0,30 et 0,80 mètre et quasiment alignées selon un axe nord-est/sud-ouest, parallèlement à un petit fossé (F5) qui s'interrompt au nord-est, à la hauteur de la troisième fosse (fig. 2). Faute de mobilier, la datation de ce fossé reste incertaine, mais un faisceau d'éléments plaide pour son ancienneté. En effet, outre la présence d'un fragment de meule en granite dans son comblement, son creusement n'apparaissait pas dans les limons supérieurs, contrairement à deux fossés de parcellaire récent (F6 et F7). De plus, un autre petit tronçon de fossé (F4) perpendiculaire à F5, était lui-même recoupé par la fosse F1. La disposition des trois fosses par rapport au fossé F5 peut laisser penser qu'elles ont pu être installées le long d'un éventuel talus qui le longeait.

Les trois fosses sont apparues après décapage de la terre végétale et du limon supérieur. Elles ont été creusées dans le limon inférieur et le substrat argileux. 


\section{La fosse à résidus de combustion (F1)}

\section{Description}

La première fosse, de forme sub-rectangulaire et à fond plat, mesure 0,60 par 0,70 mètre et n'est conservée que sur une profondeur de 0,20 mètre (fig. 3). Son comblement argilo-sableux gris, mêlé à des charbons de bois et de la cendre, a livré quelques fragments de céramique et de verre, portant des traces d'ustion différentielle, ainsi que deux petits galets de quartz ovoïdes et 33,2 g d'esquilles osseuses brûlées dont la nature, humaine ou animale, n'a pu être déterminée.

Parmi les charbons de bois prélevés, l'analyse anthracologique a permis de reconnaître la présence de chêne ou de hêtre (Archéolabs réf. ARC02/D2556B2).

\section{Mobilier (J. Pascal)}

\section{Céramique}

Aucun des huit fragments (après recollage) correspondant à six individus n’a pu être dessiné. Toutefois, les deux éléments les plus caractéristiques, gourde à décor moulé (cf. infra, fig. 14, $\mathrm{n}^{\circ} 1,1051 \mathrm{CE} 001$ ) et coupelle à décor de picots (cf. infra, fig. 14, $\mathrm{n}^{\circ}$ 2, $1051 \mathrm{CE} 002$ ), proviennent d'individus présents dans l'US 1 de la fosse F3 (cf. infra).
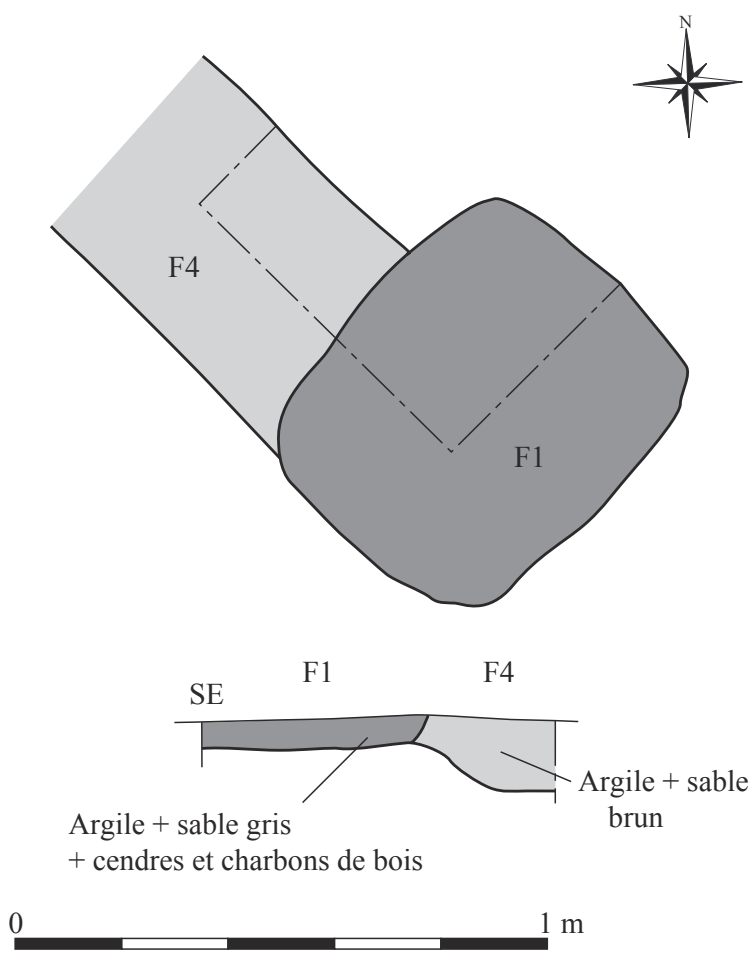

Figure 3 : Gorges, "Le Marais " : plan et coupe de la fosse F1. $\mathrm{DAO}: \mathrm{J}$. Cornec.

Figure 3: Plan and section of the pit F1.

\section{Verrerie}

Parmi les neuf fragments de verrerie, pour la plupart fracturés, déformés ou fondus par l'action du feu, correspondant à sept individus, quelques-uns ont pu être dessinés ou identifiés.

- Partie inférieure et raccord sur l'épaule d'une anse filiforme en verre bleuté provenant vraisemblablement d'une petite amphorette (fig. 4, $\mathrm{n}^{\circ} 1,1051$ VR 002).

- Fragment de fond à pied annulaire creux, verre bleuté à paroi mince (fig. 4, $\mathrm{n}^{\circ} 2,1051 \mathrm{VR} 003$ ).

- Fragment de fond à pied annulaire creux et anneau de renfort, verre légèrement verdâtre à paroi mince (fig. $4, \mathrm{n}^{\circ} 3$, 1051 VR 004).

- Fragment de fond provenant probablement d'un vase à long col tubulaire et panse hémisphérique à fond plat, verre bleuté à paroi épaisse (105 1 VR 005).

De même que pour la céramique, plusieurs fragments correspondent à des individus présents dans l'US 1 de la fosse F3 : 105 1 VR 001 recolle avec 1053 VR 001, 1051 VR 004 est proche de 1053 VR 003 et 1051 VR 005 de 1053 VR 004.

Les récipients identifiés, en verre et en céramique, correspondent à des individus de petite taille.

Mobilier lithique

Deux petits galets roulés (fig. 4, n ${ }^{\circ} 4$ et 5, 1051 LT 001), probablement en quartz, $4: 4,5 \mathrm{~g}, 16,0 \times 11 \mathrm{~mm} ; 5: 4,0$ g, $16,2 \times 10,8 \mathrm{~mm}$.

\section{Les fosses avec dépôt secondaire de crémation}

Les dépôts secondaires de crémation qui composent cet ensemble funéraire se rapportent à des structures autres que le lieu de crémation et dans lesquelles ont été placés les vestiges de la crémation prélevés sur le bûcher; il s'agit dans les deux cas de tombes où repose un vase ossuaire (dénomination d'après Blaizot et Tranoy 2004 : 171-172; Blaizot et al., 2009 : 175).

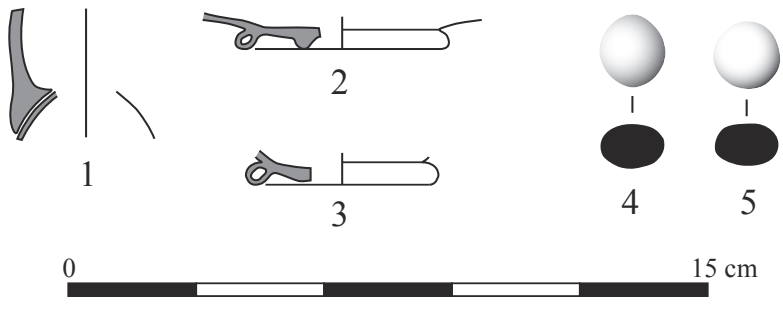

Figure 4 : Gorges, "Le Marais " : mobilier de la fosse F1. DAO : J. Cornec.

Figure 4: Artefacts of the pit F1. 


\section{La sépulture F2}

\section{Description (fig. 5)}

Dans cette sépulture, d'une profondeur conservée de 0,20 mètre, de forme sub-circulaire $(0,50$ mètre de diamètre) et à profil légèrement concave, un petit vase modelé de $7,5 \mathrm{~cm}$ de haut utilisé comme vase ossuaire était couché quelques centimètres au dessus du fond, centré dans le au milieu du creusement. Au vu de sa petite taille, il est possible que l'urne ait basculé après enfouissement ou après désagrégation d'un éventuel contenant périssable (boîte?). Cette céramique a été en partie brisée par le godet de la pelle mécanique, laissant apparaître dans son contenu des esquilles d'os brûlés. Près du vase, une vingtaine de clous ou fragments de clous en fer de diverses dimensions étaient regroupés parallèlement au flanc est de la céramique. Là encore, un sédiment charbonneux et cendreux (US 2), mais sans ossement, comblait la plus grande partie centrale de la fosse, formant une légère cuvette. Cette couche était nettement circonscrite au centre d'un comblement argilo-sableux très faiblement cendreux (US 1). L'analyse carpologique et anthracologique des charbons de bois indique la présence de chêne à feuilles caduques et d'un pépin de raisin (Archéolabs réf. ARCO6/R3362B1).

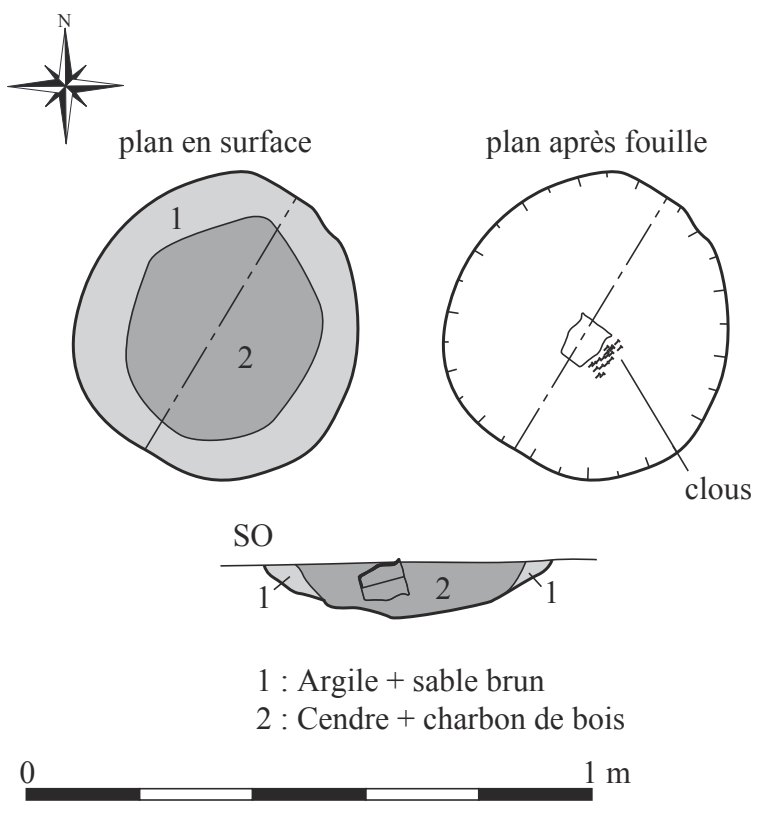

Figure 5 : Gorges, "Le Marais » : plan et coupe de la fosse F2. DAO : J. Cornec.

Figure 5: Plan and section of the pit F2.

\section{Mobilier (J. Pascal)}

\section{Céramique}

Petit pot complet (fig. 6, n 1, 1052 CE 001), modelé, brisé, fond plat très mince, panse biconique, lèvre éversée; pâte brun à gris brun, surface brune, lissée, micacée, dégraissant sableux. Pour cet objet, des parallèles peuvent être trouvés dans les nécropoles vendéennes des Landes à La Mothe-Achard (Vialet, 2010 : Sép. 26, CE 22; Sép. 68, CE 64 et Sép. 69 CE 65) et du Gardou à Saint-Georges-deMontaigu (Hervé-Monteil, 2011 : SP 1050).

\section{Métal}

Vingt clous ou fragments de clous en fer de modules divers. Certains, de petites dimensions et à tête hémisphérique, pourraient correspondre à des rivets décoratifs (fig. 6 , $\mathrm{n}^{\circ} 21$ ) ou à des clous de chaussures (fig. $6, \mathrm{n}^{\circ} 22$ et 23). D'autres, de dimensions plus importantes, sont assimilables à des clous d'assemblage pouvant avoir servi à la « construction » du bûcher (fig. 6, n ${ }^{\text {os }} 2$ à 20).

\section{Le défunt et son traitement funéraire (I. Le Goff)}

L'amas osseux est composé exclusivement de portions de calotte crânienne, dont le pariétal et le temporal (massif pétreux). Les fragments mesurent deux centimètres au maximum. D'après ces éléments, rien n'évoque la présence de plus d'un sujet. Si les quelques sept grammes de vestiges osseux offrent peu d'indices anthropologiques pour estimer précisément l'âge au décès du défunt, il s'agit assurément

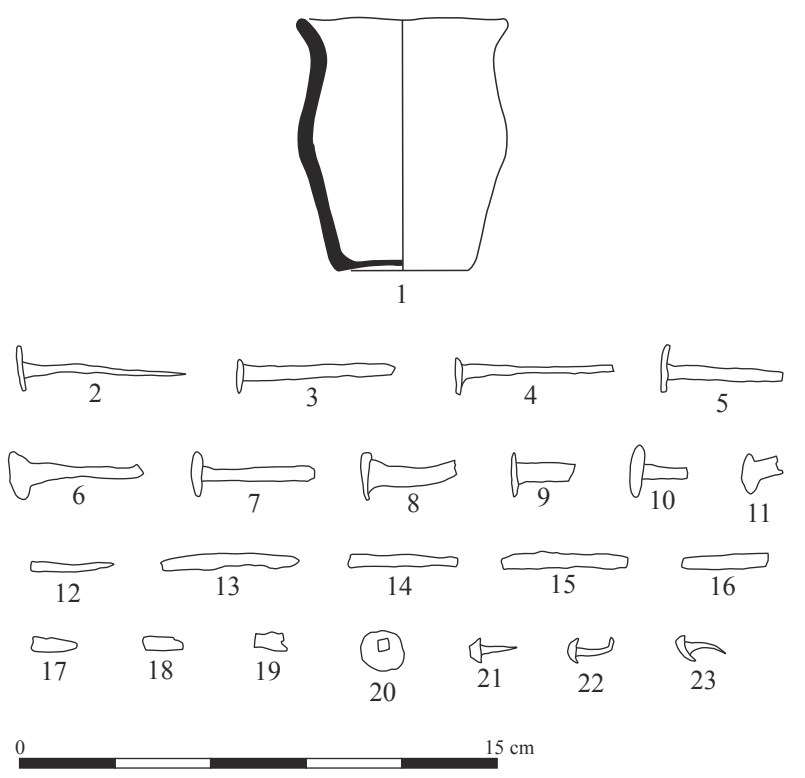

Figure 6 : Gorges, "Le Marais " : mobilier de la fosse F2. DAO : J. Cornec.

Figure 6: Artefacts of the pit F2. 
d'un sujet immature. En vue de préciser cette estimation, nous avons tiré partie de l'épaisseur de la calotte crânienne, avec les réserves d'usage quant aux conclusions, du fait de la moindre fiabilité de la méthode. Les travaux de J. Wahl proposent toutefois une intéressante corrélation entre l'épaisseur de la calotte crânienne et l'âge au décès, élaborée à partir de la collection osseuse issue de la nécropole de Süderbrarup (kr. Schleswig-Frelsburg) (Wahl, 1988). Elle représente un important corpus de cent vingt-cinq enfants et adolescents calcinés comme le fut l'enfant de la tombe F2. Leur âge est estimé d'après les méthodes classiques basées sur la maturation dentaire et osseuse.

Ainsi la moyenne $(1,7 \mathrm{~mm})$, obtenue en mesurant une dizaine de fragments sélectionnés selon les recommandations de N.-J. Gejvall, correspondrait à un âge moyen de 3 ans (intervalle de confiance : entre 2 et 6 ans) (Gejvall, 1963). D'après ces indices, les ossements concorderaient avec ceux d'un jeune enfant.

La constitution du dépôt cinéraire correspond à un assemblage simple. Il comprend un petit récipient renfermant les seuls restes osseux du défunt sans ajout d'autres types de vestiges (faune ou objet), à l'exception d'éventuels éléments putrescibles qui n'ont pas laissé de traces perceptibles. Ne s'y trouvent pas non plus les résidus de combustion du bûcher alors que sur les bûchers traditionnels, les os sont mêlés aux cendres à l'issue de la crémation.

Leur absence dans l'urne implique des gestes de collecte spécifiques qui visent à séparer les os des résidus du bûcher. Dans le vase de la tombe F2, les os sont donc les seuls témoins de la crémation qui font l'objet du dépôt, contrairement au dépôt mixte de crémation. Ces actions techniques récurrentes au cours des pratiques funéraires antiques sont de ce fait probablement porteuses de sens et contribuent à transformer la personne décédée en défunt. Toutefois, des résidus de combustion (ceux du bûcher?) ne sont pas absents de la tombe et viennent combler la fosse.

On notera l'adéquation entre les modestes dimensions du contenant sélectionné et la faible quantité des vestiges osseux. Ce choix anticipe sans doute le faible volume osseux produit par la combustion d'un squelette de jeune enfant mais également la faible masse d'os transférée dans le vase ossuaire.

Si quelques pièces osseuses éparses figurent dans le sédiment de comblement de l'urne, la plupart d'entre elles, regroupées au fond du récipient, forment un amas compact de quatre à cinq centimètres d'épaisseur (fig. 7). Sa morphologie ne plaide pas en faveur de l'usage d'une enveloppe organique comme, par exemple, un linge maintenant les os avant leur introduction dans le pot. Le tas d'os se développe non pas dans le fond de l'urne mais dans la panse, en cohérence avec la position couchée du contenant, ce qui

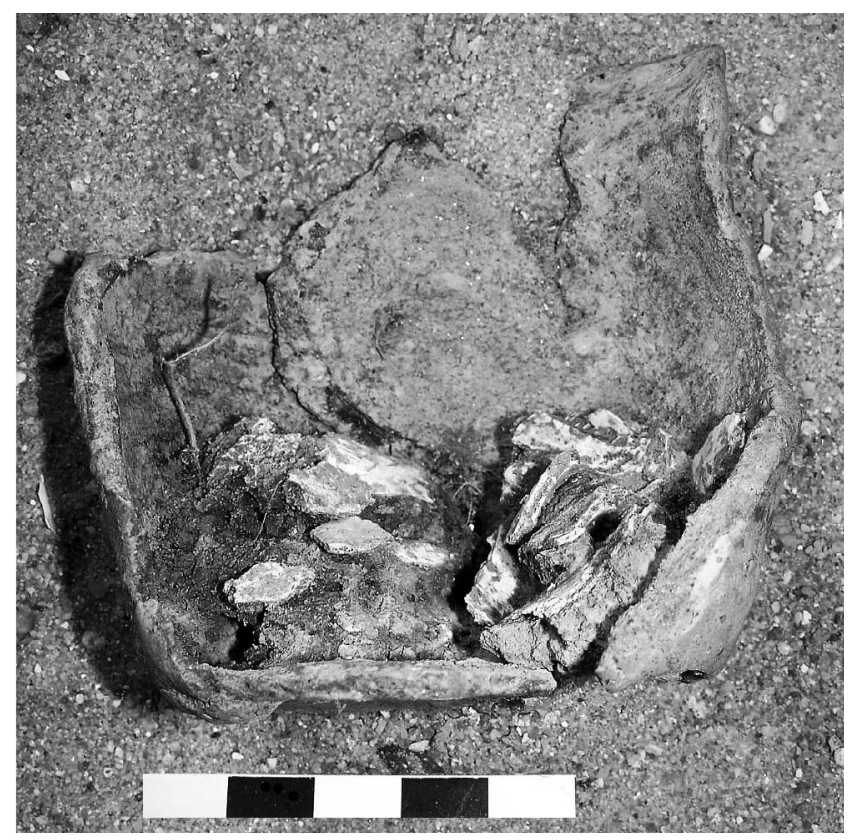

Figure 7 : Gorges, "Le Marais " : amas osseux dans l'urne de la fosse F2. On observe un glissement du dépôt osseux dans la panse du vase, situation cohérente avec celle du contenant. Cliché : I. Le Goff.

Figure 7: Osseous heap in the urn of the pit F2. We observe a sliding of the osseous deposit in the paunch of the vase, the coherent situation with that of the packaging.

indiquerait un glissement des os dans le creux de la panse avant colmatage du vase ossuaire par la terre. Basculement involontaire ou position souhaitée par les opérateurs, l'analyse de cette situation doit tenir compte d'un autre élément. Bien que l'urne se trouve prise dans le comblement charbonneux de la tombe (US 2), c'est un sédiment différent, de couleur jaune, qui enrobe les os et remplit le récipient. On se demande si le comblement ne s'est pas effectué ailleurs ou avant le dépôt de l'US 2.

\section{La sépulture F3 (fig. 8 et 9)}

\section{Description de la structure}

D'une profondeur conservée de 0,60 mètre, de forme ovale et mesurant 0,50 par 0,60 mètre, la troisième fosse présentait un remplissage plus complexe. Au centre de la fosse, une céramique commune complète était disposée verticalement à quelques centimètres du fond, ce qui suppose un support en matériau périssable. Les radiographies (fig. 10) et la fouille de cette céramique ont révélé la présence, à l'intérieur, d'une coupe en verre remplie d'esquilles d'os. Une seconde coupe, en verre également, posée à l'envers sur la première en constituait le couvercle. Directement au sud-ouest du vase réceptacle, à environ 5 centimètres du 

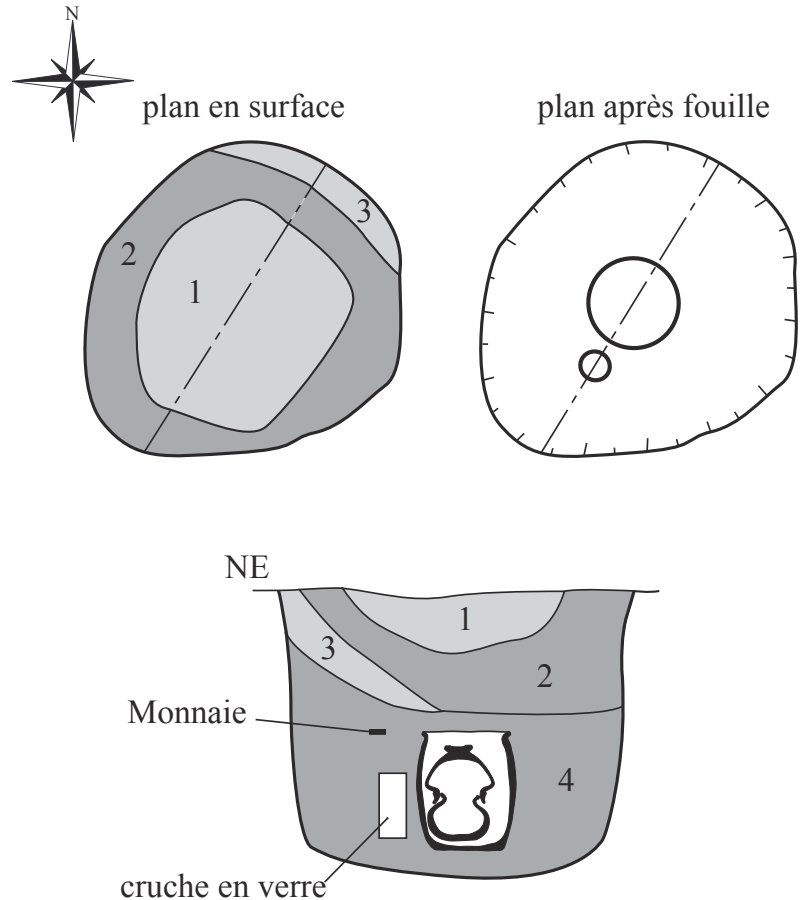

$1:$ Amas d'objets brisés et brûlés

$2:$ Cendres et charbons de bois

3: Argile jaune

$4:$ Cendres et charbons de bois

0 $1 \mathrm{~m}$

Figure 8 : Gorges, "Le Marais " : plan et coupe de la fosse F3. $\mathrm{DAO}: \mathrm{J}$. Cornec.

Figure 8: Plan and section of the pit F3. fond, reposait une cruche en verre brisée. Ces éléments étaient noyés dans un sédiment extrêmement charbonneux et cendreux (US 4), dans la partie supérieure duquel une monnaie en bronze a été découverte, à plat. Deux fragments de ferrures (?) proviennent du même niveau mais aucune esquille d'os.

Un fin niveau d'argile jaune (US 3), partant du bord nord-est de la fosse et en pendage vers le centre, séparait le remplissage inférieur d'un sédiment lui aussi charbonneux (US 2) qui a livré uniquement un fragment de fibule ou de parure en bronze.

L'analyse anthracologique (Archéolabs réf. ARCO6/ R3362B1) des charbons de bois de l'US 2 indique la présence de chêne à feuilles caduques ( 27 fragments), d'aulne ou de noisetier (2 fragments) et d'orme (1 fragment). Un grain de blé a également pu être identifié.

En surface et au centre de la fosse, un agglomérat, composé de nombreux fragments de céramiques et de verreries, d'une figurine de Vénus Anadyomène brisée, d'une petite perle en verre et de sept petits galets de quartz roulés de forme ovoïde, formait une lentille d'une quinzaine de centimètres d'épaisseur (US 1) dont l'aspect relativement régulier et concentré suggère l'existence d'un contenant en matière périssable (sac en toile ou panier?). Tous ces éléments, mêlés à de l'argile beige, avaient subi l'action du feu (fig. 11).

\section{Le mobilier (J. Pascal)}

\section{US 4}

Céramique - Réceptacle : grand pot à fond plat, panse sub-cylindrique légèrement renflée, lèvre éversée; céramique grossière, modelée, irrégulière, décolorée et épaufrée par le

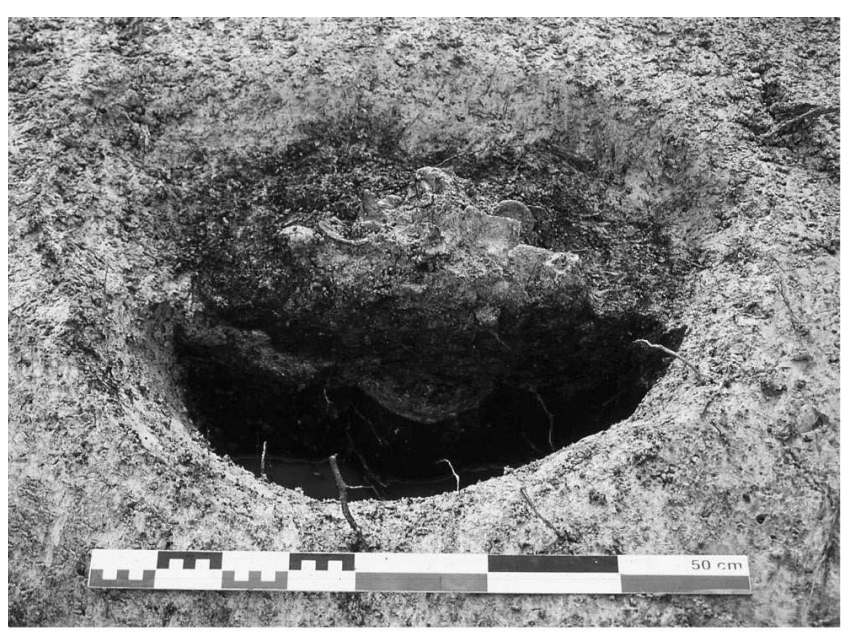

1

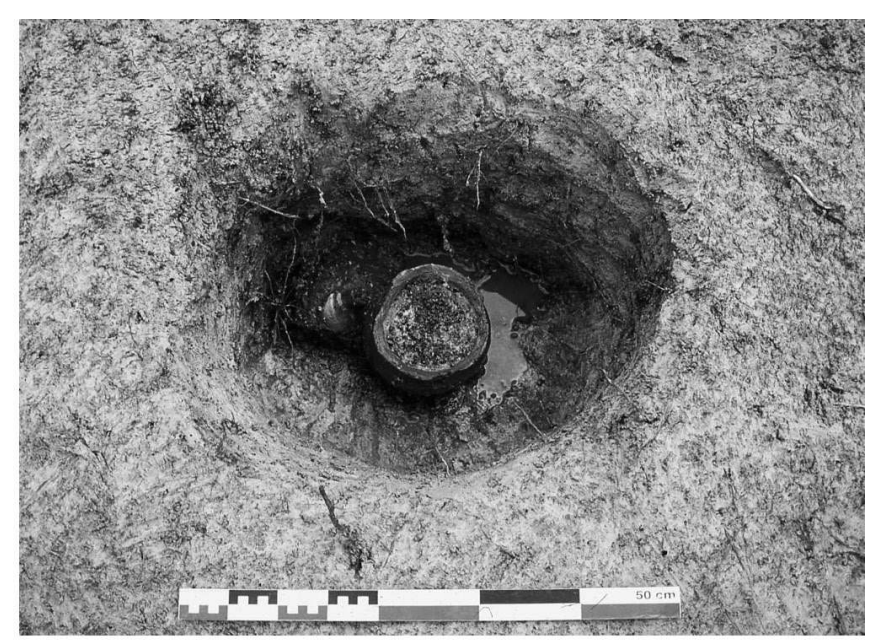

2

Figure 9 : Gorges, «Le Marais » : clichés de la fosse F3. Clichés : P. Bellanger.

Figure 9: Pictures of the pit F3. 


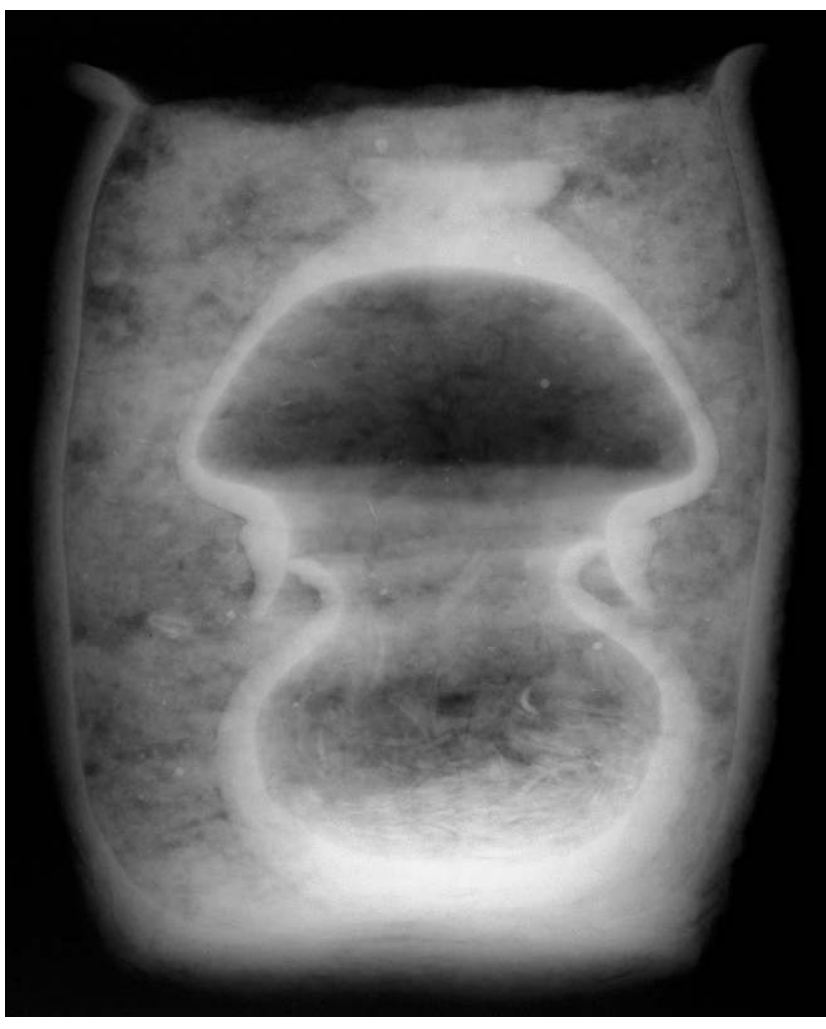

Figure 10 : Gorges, «Le Marais » : radiographie de l'urne contenue dans la fosse F3. Cliché : Laboratoire Arc'Antic.

Figure 10: Radiography of the urn contained in the pit F3.

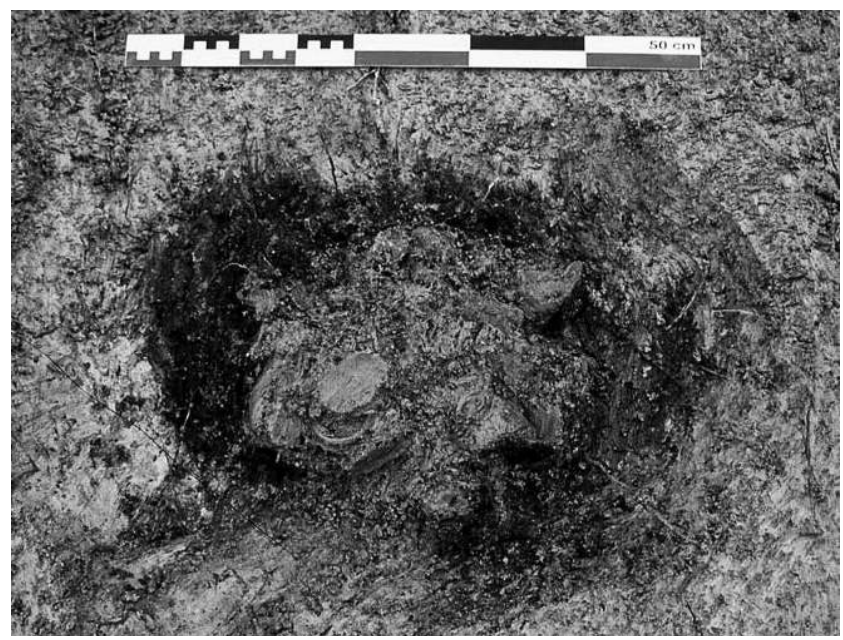

Figure 11 : Gorges, "Le Marais " : amas d'objets brisés et brûlés en surface de la fosse F3. Cliché : P. Bellanger.

Figure 11: Heap of broken and burned artefacts on the top of the pit F3.

contact avec les cendres (fig. 12, $\mathrm{n}^{\circ} 1,1053 \mathrm{CE} 016$ ). Objet complet contenant le vase ossuaire VR 008 et son couvercle VR 007. Ici peuvent également être cités des parallèles avec la nécropole des Landes (Vialet, 2010 : Sép. 11 CE 11 et Sép. 33 CE 29), ainsi qu'avec celle du Gardou (HervéMonteil, 2011 : SP 1049); les musées de Vendée conservent un vase similaire issu de la nécropole de La Créancière (commune de Dompierre-sur-Yon), fouillée en 1854 (information É. Bernard $\dagger$ ).

Dessin réalisé d'après radiographie
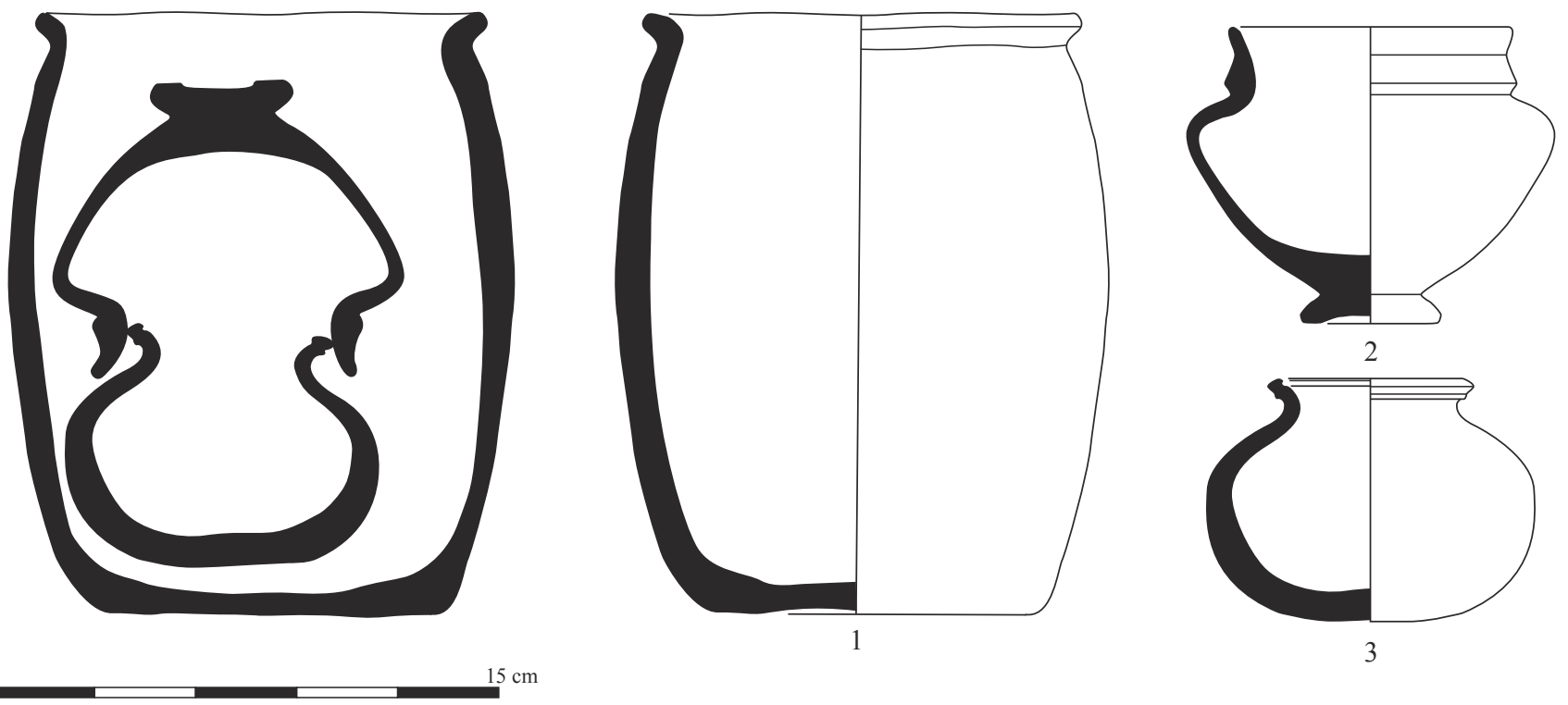

2

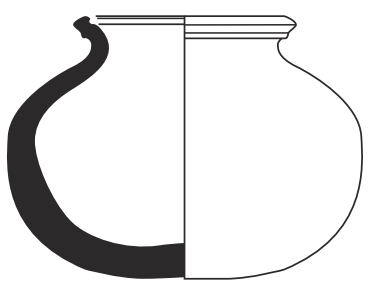

3

Figure 12 : Gorges, "Le Marais " : mobilier de la fosse F3 : l'ossuaire, le couvercle et le réceptacle. DAO : J. Cornec. Figure 12: Artefacts of the pit F3: The ossuary, the lid and the receptacle. 
Verre ossuaire - Vase en verre à panse globulaire, col resserré, lèvre éversée (fig. 12, n 3, 1053 VR 008). L'état de conservation du vase et le fait qu'il se soit défait en micro éclats, malgré sa consolidation, lors de la fouille du dépôt osseux n'autorisent pas une identification fine, l'observation de la lèvre sur la radiographie semble permettre un rapprochement avec la forme Isings $67 \mathrm{c}$, ou, éventuellement, la forme Isings 94.

Couvercle du verre ossuaire - Vase en verre de type Isings $67 \mathrm{~b}$ contenu dans le vase $1053 \mathrm{CE} 016$, renversé, servant de couvercle au vase 1053 VR 008, complet et intact (fig. 12, no 2, 1053 VR 007). Vase à pied annulaire, panse hémisphérique, épaule surbaissée bien marquée, col resserré, lèvre roulée vers l'extérieur, formant un bandeau mouluré, verre verdâtre. Cette forme très courante est fréquemment utilisée comme vase ossuaire; on peut là encore citer les nécropoles des Landes (Vialet, 2010 : Sép. 19, VR 5) et du Gardou (Hervé-Monteil, 2011 : SP 1122/VP 1123).

Dépôt associé au réceptacle - Carafe de type Isings $88 \mathrm{~b}$, panse sphérique, fond aplati, col tubulaire évasé et bec tréflé (fig. 13, 1053 VR 006). Verre incolore à paroi mince, décor de quatre incisions horizontales au tiers inférieur de la panse. Objet déposé incomplet dans la fosse, l'anse et la quasi-totalité de la lèvre manquent.

Autres mobiliers contenus dans l'US 4 - Une monnaie de bronze, de $22 \mathrm{~mm}$ et pesant $3,13 \mathrm{~g}$, illisible (105 3 MT 003). Plusieurs fragments indéterminés en fer (dont deux éléments de ferrure?; 1053 MT 004).

\section{US 2}

Métal - Élément indéterminé en bronze (il s'agit probablement d'une fibule ou d'un fragment de fibule; 1053 MT 001).

- Élément indéterminé en fer (105 3 MT 002).

\section{US 1}

Céramique (identification des sigillées : R. Delage) Plusieurs fragments d'une gourde à décor moulé de type Déchelette 63 (Vertet 63), panse cylindrique aplatie, modelée en deux parties dans un moule à décor en creux, col court, cylindrique et rapporté, lèvre éversée, communication entre la panse et le col percée avec un clou (fig. 14, $\mathrm{n}^{\circ} 1$, 1053 CE 006); décor peu lisible semblant s'organiser en cercles concentriques de motifs répétés, un seul est actuellement identifié : une " corne " striée; pâte fine, gris beige, brûlée, engobe vraisemblablement plombifère. Même individu que 1051 CE 001.

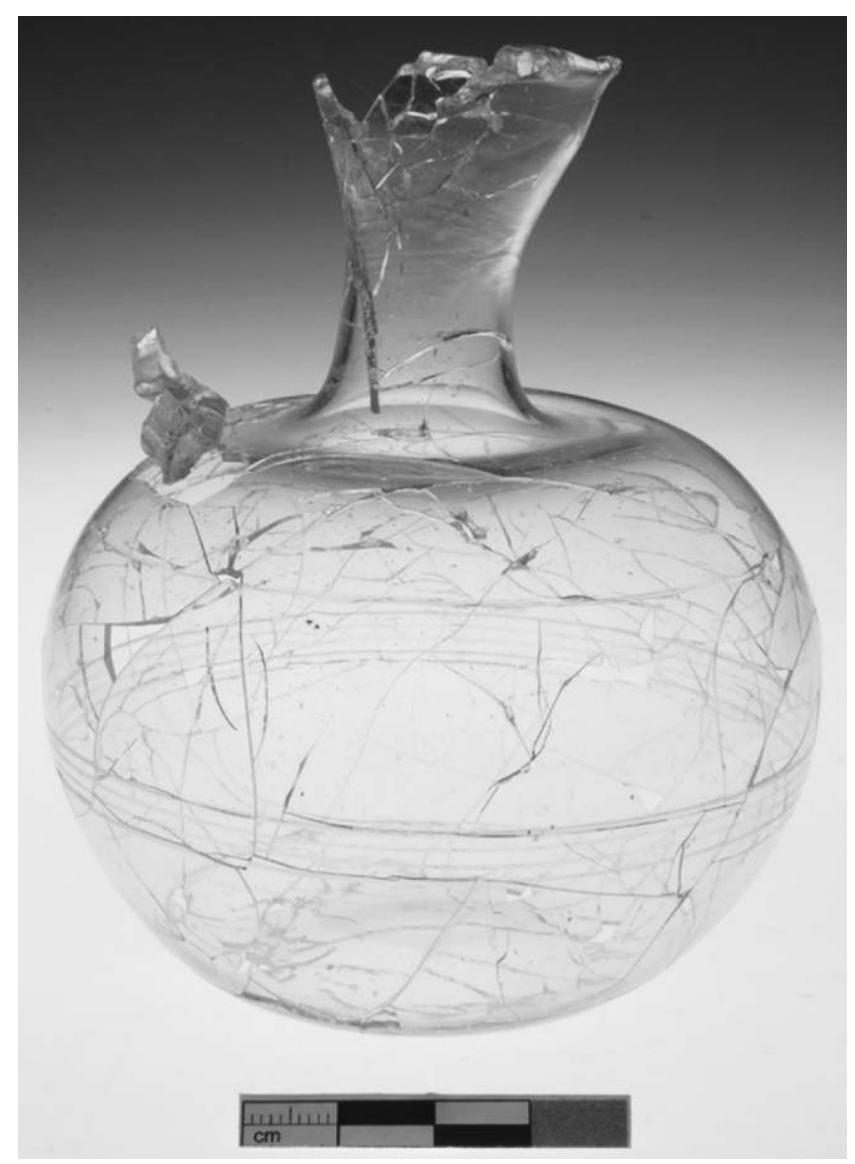

Figure 13 : Gorges, "Le Marais " : mobilier de la fosse F3 : la cruche en verre. DAO : J. Cornec.

Figure 13: Artefacts of the pit F3: the glass jar.

Petite coupe tripode, fond plat, panse hémisphérique, lèvre, amincie, un groupe de picots rapportés sous la lèvre sert de préhension (fig. 14, n 2, 1053 CE 014); pâte semifine, brûlée, surface micacée, dégraissant sableux. Même individu que 1051 CE 002.

Coupelle en sigillée de forme Drag. 38, coupelle hémisphérique à collerette de préhension peu retombante, lèvre mince moulurée pour dégager un petit bourrelet (fig. 14, $n^{\circ} 3,1053$ CE 002); pâte fine, brûlée, profil restitué à partir de fragments non jointifs, probablement Gaule du Centre.

Coupelle en sigillée de forme Ve D2 (Drag. 42/Ritt. 14B), lèvre rabattue à l'horizontale (fig. 14, $\mathrm{n}^{\circ} 4,1053 \mathrm{CE} 001$ ), ornée de « feuilles d'eau » à la barbotine, bord mouluré pour former un bourrelet annulaire sur lequel viennent se greffer deux anses plates; pâte fine, brûlée, Sud Gaule, probablement La Graufesenque.

Coupelle en sigillée de forme Ve C1 (Drag. 46), lèvre formant un bourrelet annulaire, parois minces (fig. 14, $\mathrm{n}^{\circ} 5$, 1053 CE 003); pâte fine, brûlée, profil restitué à partir de fragments non jointifs, probablement Sud Gaule. 
Coupelle en sigillée de forme Bet 31 (Walters 80), bord vertical, lèvre soulignée par une cannelure pour dégager un léger bourrelet (fig. 14, $\mathrm{n}^{\circ}$ 6, $1053 \mathrm{CE}$ 004), la finesse des parois et le mauvais état de conservation des fragments ne laissent pas apparaître les ressauts caractéristiques de la forme à la jonction fond-bord; pâte fine, brûlée, Gaule du Centre.

Neuf fragments d'un vase biconique, pied cylindrique mouluré, carène marquée par un large replat vertical, lèvre éversée soulignée par une cannelure (fig. 14, $\mathrm{n}^{\circ} 7$, $1053 \mathrm{CE}$ 015); pâte gris noir, brûlée, surface lustrée. Il s'agit d'un exemplaire de petit module d'une forme très courante, produite notamment dans les ateliers du CentreOuest (Thésée-Pouillé); des exemplaires de plus grande taille ont servi d'ossuaire dans la nécropole du Gardou (HervéMonteil, 2011 : SP 1008, 1031, 1057).

Six fragments provenant vraisemblablement d'une même petite amphorette en céramique semi-fine, fond plat, panse ovoïde, épaule bien marquée, col étroit, parois minces (fig. 14, no 8, 1053 CE 011); pâte gris beige, brûlée.

Partie inférieure d'un gobelet en céramique fine (ou métallescente) de type Bet 312 ("gobelet flûte »), pied annulaire largement débordant, mouluré, probablement rapporté sur une base étroite, creuse (fig. 14, n 9, 1053 CE 009); pâte fine, brûlée, engobe noir, Gaule du Centre.

Partie inférieure d'un gobelet en céramique fine, petit pied cylindrique mouluré, fond épais (fig. 14, $\mathrm{n}^{\circ} 10$, 1053 CE 007); pâte fine, brûlée, engobe noir.

Partie inférieure d'un gobelet en céramique fine, petit pied tronconique (fig. 14, nº 11, $1053 \mathrm{CE} 008$ ) ; pâte fine, brûlée, engobe noir.

Fond de coupelle en sigillée de forme Bet 44, pied mouluré, élément vraisemblablement retaillé volontairement pour une utilisation secondaire (fig. 14, $\mathrm{n}^{\circ} 12$, 1053 CE 005); pâte fine, brûlée, probablement Gaule du Centre.

Pied annulaire largement débordant d'un gobelet proche du gobelet $n^{\circ} 9$ (fig. 14, n 13, 1053 CE 010); pâte fine, brûlée, engobe noir.

\section{Verrerie}

Quatre fragments d'un vase en verre bleu foncé (fig. 14, $\mathrm{n}^{\circ}$ 14, 1053 VR 001), localement brûlé et déformé, fond à ombilic, partie inférieure de la panse hémisphérique, marquée par une incision horizontale.

Petite perle tubulaire à section hexagonale (fig. 14, $\mathrm{n}^{\circ} 15$, 1053 VR 002), verre vert.

Outre la perle, seule une faible proportion des nombreux fragments de verreries, pour la plupart fracturés, déformés ou fondus par l'action du feu du bûcher, a pu être identifiée, la plupart correspond à des individus ou à des types présents dans la fosse F1 (cf. supra) : 1053 VR 001 recolle avec 1051 VR 001; 1053 VR 003, en verre légèrement verdâtre et à paroi mince est proche de 1051 VR 004 et 1053 VR 004, en verre bleuté et à paroi épaisse, pourrait provenir du même récipient que 1051 VR 005.

Figurine - Figurine en terre blanche (fig. 14, $\mathrm{n}^{\circ}$ 16, 1053 TC 018), moulée, Vénus sortant du bain (Anadyomène), tenant sa chevelure de la main droite et une draperie de la main gauche, type courant (Herbert, 1995 : $\mathrm{n}^{\circ} 3$; Rouvier-Jeanlin, $1972: \mathrm{n}^{\text {os }} 36$ et 38). Les pieds et la base manquent, reliefs usés et empâtés (issus d'un surmoulage?), la face postérieure semble plus soignée, notamment le traitement de la chevelure; pâte blanchâtre, fine, brûlée.

Mobilier lithique - Sept petits galets roulés, matériaux divers (fig. 14, $\mathrm{n}^{\text {os }} 17$ à 23, 1053 LT 001), $17: 11 \mathrm{~g}, 24,0 \mathrm{x}$ $17,8 \mathrm{~mm} ; 18: 12 \mathrm{~g}, 23,4 \times 17,5 \mathrm{~mm} ; 19: 8 \mathrm{~g}, 20,0 \mathrm{x}$ $16,5 \mathrm{~mm}$, irrégulier; $20: 7 \mathrm{~g}, 19,4 \times 13,2 \mathrm{~mm} ; 21: 5 \mathrm{~g}$, $16,1 \times 11,4 \mathrm{~mm} ; 6: 5 \mathrm{~g}, 16,7 \times 12,9 \mathrm{~mm}$, irrégulier; 22 : $2,5 \mathrm{~g}, 13,3 \times 11,0 \mathrm{~mm}$.

\section{Le défunt et son traitement funéraire (I. Le Goff)}

Le vase ossuaire de la fosse F3 renfermait également les restes $(40,7 \mathrm{~g})$ d'un sujet immature. Il est âgé entre 2 et 3 ans selon la maturation de ses dents (Moorrees et al., 1963). L'épaisseur de la calotte, avec une moyenne de $1,8 \mathrm{~mm}$, présente un intervalle de confiance étendu qui élargit l'estimation de l'âge possible du défunt. Il est estimé entre 3 et 6 ans (moyenne à 5 ans). La corrélation entre les stades de calcification dentaire et l'âge calendaire étant mieux connue, ce sont les résultats de cette méthode que nous retiendrons. D'autres indices anthropologiques, comme la maturité des os longs, ne sont pas disponibles.

Lélaboration de ce dépôt osseux s'avère plus complexe que dans le cas précédent. Constitué dans le même esprit que celui de la fosse F2, il est destiné également à un unique sujet, et se caractérise par la sélection d'un contenant de petite dimension où sont déposés les seuls os du défunt. N'y figurent ni résidus de combustion, ni mobilier. L'urne ne reçoit qu'une partie des vestiges du squelette : les $40,7 \mathrm{~g}$ d'os concernent surtout les os de la tête (22,6 g) abondamment représentés ( $55,5 \%$ de l'ensemble). S'ajoutent quelques portions d'os longs $(8,8 \mathrm{~g}-21,6 \%)$. Le taux d'identification s'élève quasi à $78 \%$ des os.

La complexité de ce dépôt résulte de l'association de quatre éléments. Un pot en verre, retourné en guise de couvercle, s'ajuste sur l'embouchure de l'urne ainsi hermétiquement fermée, empêchant toute infiltration de terre (fig. 15, $\mathrm{n}^{\circ} 1$ ). Le réceptacle en terre, aux dimensions ajustées, reçoit cet assemblage dont l'introduction n'a pu s'effectuer avec les deux mains, faute d'espace (fig. $15, \mathrm{n}^{\circ} 2$ ). Un quatrième 

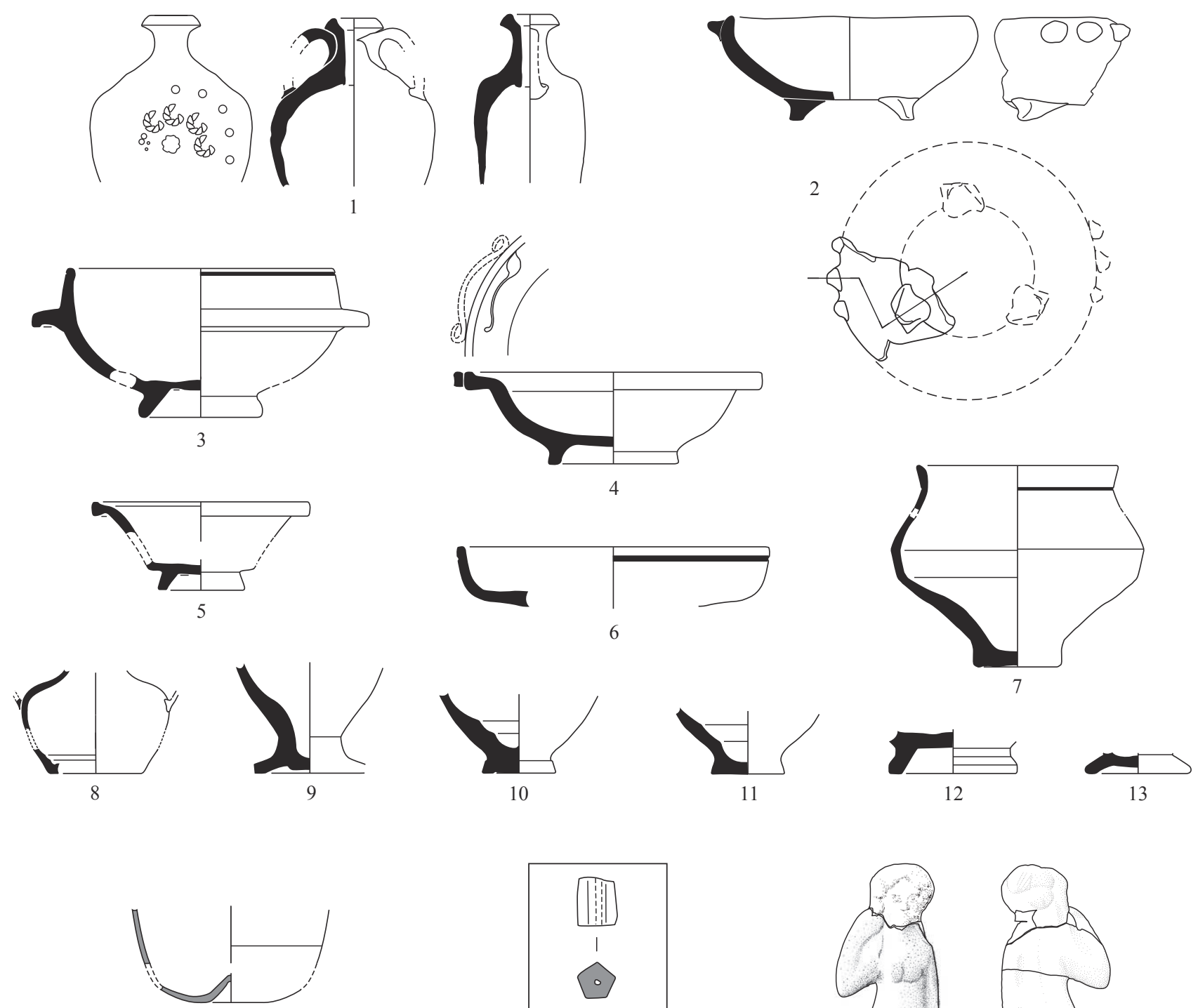

14
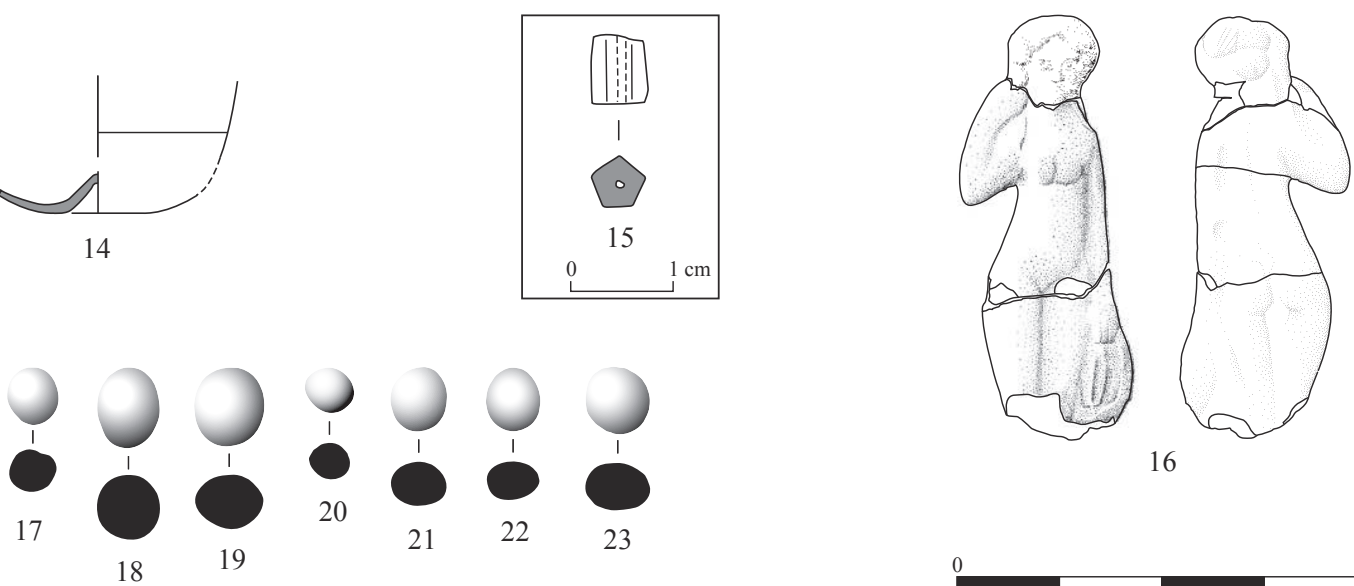

23

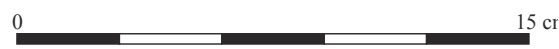

Figure 14 : Gorges, "Le Marais » : mobilier de la fosse F3 : objets brisés et brûlés issus de l'amas de l'US1. DAO : J. Cornec.

Figure 14: Artefacts of the pit F3: broken and burned artefacts from the heap of the US1.

élément participe sans doute à la constitution de l'urne. Il en subsiste quelques traces au niveau du col de la céramique : y figurent des morceaux de bois noircis, pris dans un sédiment limono-argileux de couleur jaune (couche 1; fig. 15, $\mathrm{n}^{\circ} 3$ ). Ils se localisent également plus en profondeur dans le récipient, jusqu'à l'épaulement de l'urne. Là, se développe un sédiment pulvérulent blanc (couche 2) qui évoque une fine boue; de l'eau aurait stagné dans l'urne recouvrant de boue le tiers supérieur de celle-ci (fig. 16). Plus bas encore, les fragments, plus petits, se raréfient (couches 3 et 4). 


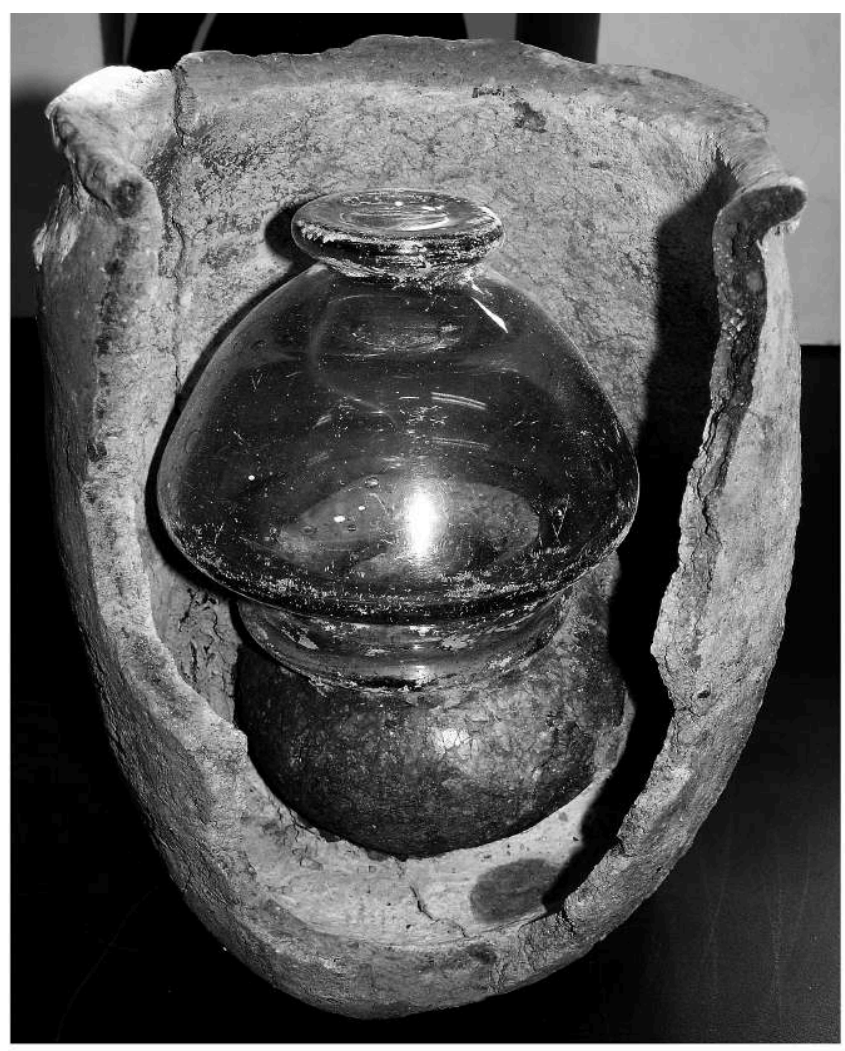

1

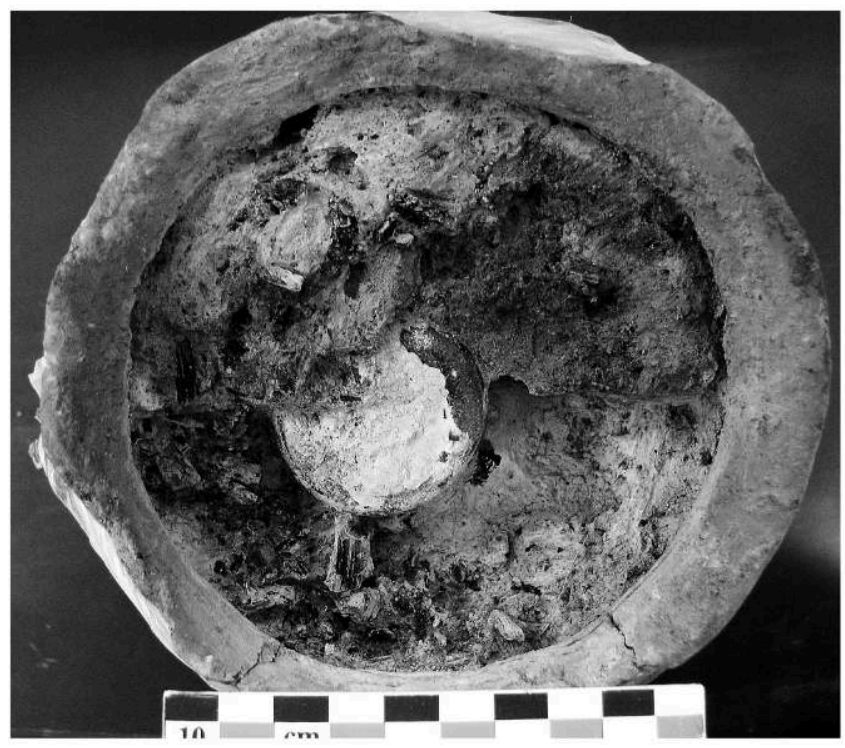

3

Le matériau noirci correspondrait à du bois décomposé plutôt qu'à du charbon. Sa localisation dans le tiers supérieur du vase en terre, sans doute vide un certain un temps

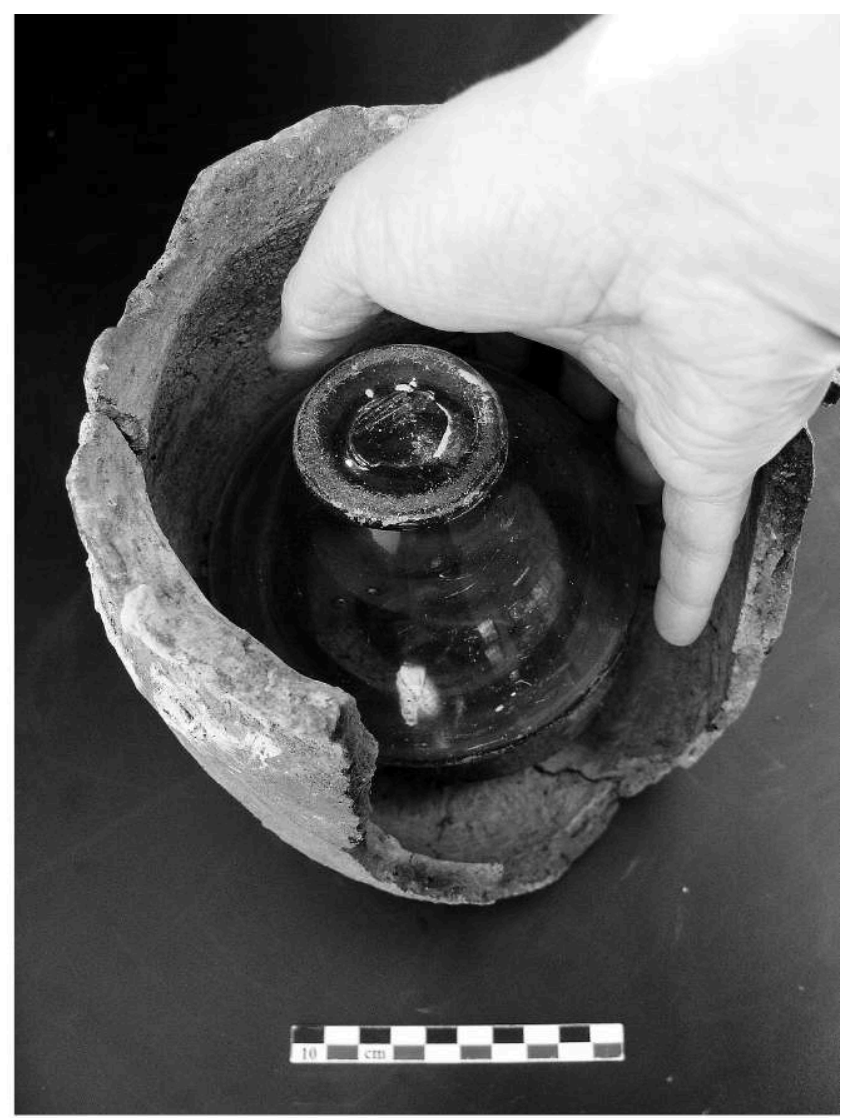

2

Figure 15 : Gorges, "Le Marais " : fouille de l'ossuaire de la fosse F3. Clichés : I. Le Goff. 1 : assemblage des trois récipients qui constitue le dépôt cinéraire placé dans la tombe. 2 : assemblage des deux récipients en verre placés dans un pot en terre ajusté à leurs dimensions. 3 : vue en plan du comblement du réceptacle en terre. Le tiers supérieur se caractérise par un sédiment comportant de nombreux morceaux de bois désagrégé (couche 1) qui se répartissent de part et d'autre du vase couvercle. Ils sont interprétés comme les résidus de décomposition d'un élément en bois placé sur l'embouchure du réceptacle.

Figure 15: Ossuary's search of the pit F3. 1: assembly of three containers which establishes the funerary deposit placed in the grave. 2: assembly of the two glass containers placed in a clay pot fitted to their size. 3: Plan view of the filling of the clay pot. The superior part is characterized by sediment containing numerous pieces of disintegrated wood (stratum 1) on all sides of the vase lid. They are interpreted as the residues of decomposition of a wooden element placed on the mouth of the receptacle.

comme l'indiquent les signes de stagnation d'eau, évoque un couvercle qui s'effrite. 


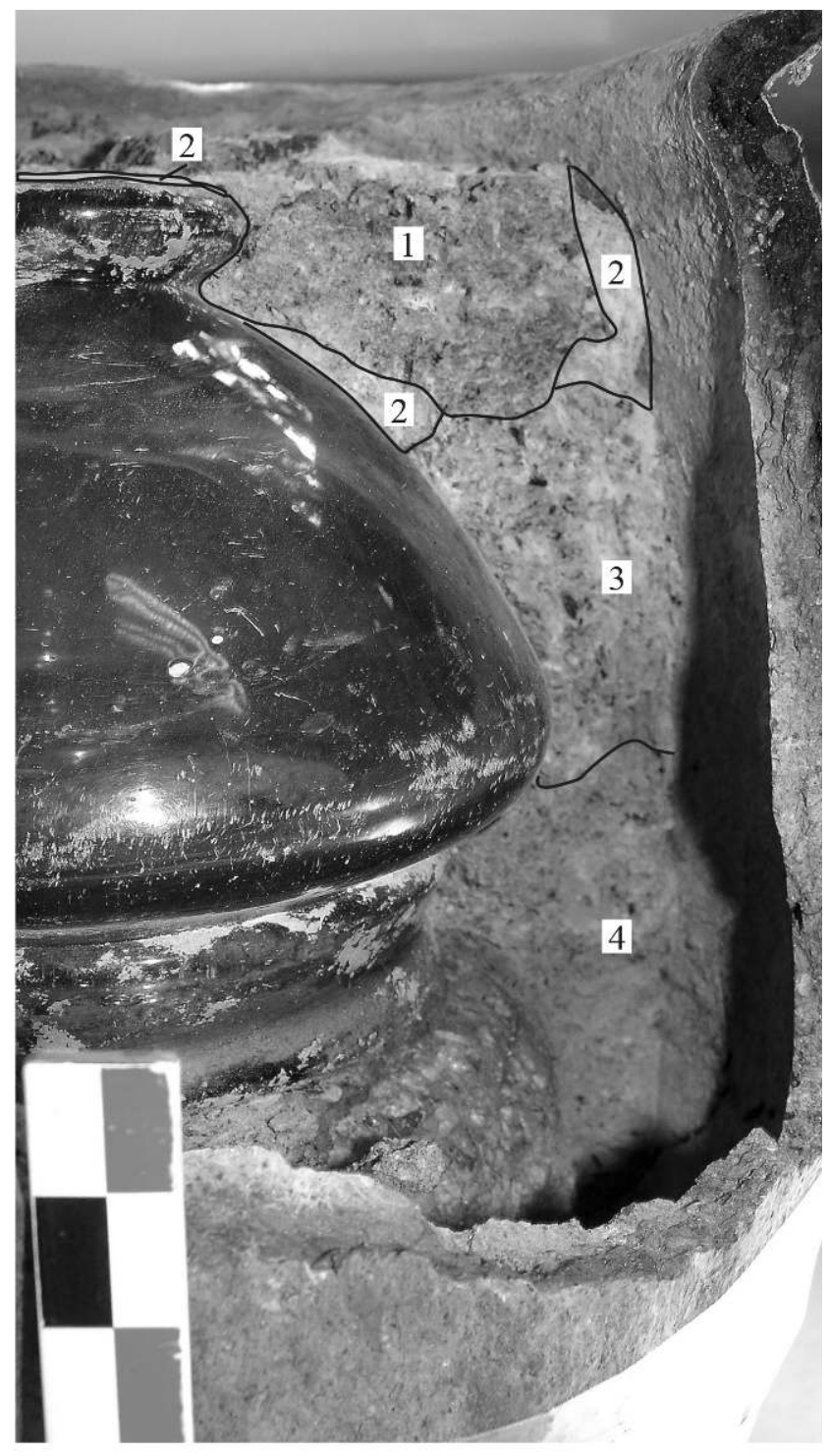

1: Sédiment brun jaune avec des nodules d'oxyde de fer, des particules et de nombreux morceaux de charbon.

2 : Sédiment pulvérulent, blanc, sans charbon ni inclusion.

3 : Sédiment issu d'un mélange des deux couches précédentes.

4 : Sédiment gris clair avec particules de charbon et quelques fragments de charbon

Figure 16 : Gorges, "Le Marais » : distribution des morceaux de charbon de bois dans l'urne de la fosse F3.

Figure 16: Distribution of the pieces of charcoal in the urn of the pit F3.

\section{Crémation du défunt et ENSEVElissement DES OS (I. LE GOFF)}

\section{Le dénombrement des défunts}

Au sein de chacune des tombes, le nombre minimum d'individus (NMI) s'élève à un. Les données ostéologiques (âge au décès, pièce en double) n'indiquent pas la présence de plus d'un défunt par tombe. Chacune contient les vestiges d'un squelette immature : un enfant décédé entre 2 et 3 ans pour le défunt de la tombe F3; un enfant également décédé jeune, dans la tombe F2, comme l'indique l'épaisseur de sa voûte crânienne proche de celle du sujet de la sépulture F3.

Dans la fosse F1, composée surtout de résidus de combustion, les quelques esquilles osseuses restées indéterminées ne contribuent pas au dénombrement des défunts.

L'absence de collage entre les ensembles osseux des tombes $\mathrm{F} 2$ et $\mathrm{F} 3$ tend à infirmer l'hypothèse d'une distribution des os d'un individu dans deux structures distinctes.

\section{La quantité et la nature des os ensevelis}

Dans la fosse F2, ne sont ensevelies que des portions de calotte crânienne. Avec $7 \mathrm{~g}$, elles ne constituent qu'une partie du squelette céphalique. La présence exclusive d'un secteur du squelette trouve quelques explications dans les gestes de collecte ou de combustion. L'opérateur a pu rechercher et regrouper les os de la tête pour les transférer dans la tombe ou, autre hypothèse, la conduite de la crémation peu accompagnée aurait limité les actions déstructurant le squelette (Grévin, 2004; Depierre, 2014). Ainsi, la situation des morceaux du crâne, encore groupés après crémation, aurait facilité l'élaboration d'une " poignée " maintenant la cohérence anatomique jusque dans l'ossuaire.

Dans la fosse F3, le transfert des vestiges concerne $40 \mathrm{~g}$ d'os, et différents secteurs anatomiques du squelette (tête, os longs). Pour donner une idée de la part manquante, la crémation d'un garçonnet de quatre ans en four crématoire réduit le corps à $636 \mathrm{~g}$ d'os (Depierre, 2014, d'après Warren et Maples). Dans ce cas également, une partie du corps seulement figure dans l'urne avec à nouveau une forte présence des os de la tête (55,5\% de l'ensemble osseux).

\section{Le stade de crémation}

La coloration des ossements, homogène et à dominante blanche, indique une crémation menée jusqu'à disparition des chairs et calcination des os. Ceux de la tombe F2 s'avèrent exclusivement blancs et crayeux, y compris le massif pétreux gauche, secteur particulièrement épaissi de l'os 
temporal signalant une combustion suffisamment avancée pour blanchir l'intérieur de la cavité crânienne.

Dans la tombe F3, l'ensemble du corps de l'enfant présente les signes d'une calcination moindre avec des pièces osseuses qui comportent encore des plages gris foncé. Par exemple, le massif pétreux droit est resté entièrement gris foncé.

\section{Mobiliers et datation (J. Pascal)}

Sur ce site, l'identification et l'étude du mobilier se heurtent au mauvais état de conservation et à la grande fragmentation du mobilier ayant subi l'action du feu.

Compte tenu des nombreux collages inter structures, il ne fait toutefois aucun doute que le mobilier de la fosse F1 et de l'US 1 de la fosse F3 provient d'un lot unique réparti en deux dépôts distincts, cette partition est volontaire et ne résulte pas d'une perturbation du site. Il paraît vraisemblable de l'interpréter comme des vestiges de mobiliers ayant été brûlés (avec le défunt ou sur un autre foyer) et recueillis au même titre que les ossements; rien n'indique par ailleurs que le prélèvement ait été systématique.

Pour le dépôt en surface de la tombe F3 (US 1), les comptages en NMI de la céramique font apparaitre une figurine en terre blanche, quatre coupelles en sigillée, un fond, probablement retaillé, en sigillée, une gourde en céramique à vernis plombifère, quatre gobelets en céramique fine, une amphorette et une coupelle tripode en céramique « semifine ", un vase biconique en céramique à cuisson réductrice et des fragments isolés de trois individus non identifiés, en céramique commune. Soit une figurine et seize récipients dont seuls deux sont quasiment complets (trois en comptant le fond retaillé) et cinq autres reconstituables. Il s'agit essentiellement d'objets de petites dimensions, importés, au sein desquels on notera l'absence d'assiettes, de cruches et la faible proportion de céramiques communes.

L'utilisation de céramique non tournée comme vase ossuaire ou réceptacle d'urne trouve des correspondances sur deux nécropoles vendéennes, à La Guyonnière et à La Mothe-Achard (Thébaud, 2012). La permanence de ces céramiques modelées à l'époque romaine, notamment en territoire picton, est désormais bien reconnue (Guitton et al., 2015).

La verrerie est trop fragmentée pour permettre une identification ou des comptages fiables, elle devait toutefois être assez abondante (une dizaine d'individus) et diversifiée (verre bleu foncé, verre bleuté, vert verdâtre, verre décoloré). Il convient éventuellement d'ajouter à ce décompte le réceptacle, les deux urnes en verre ainsi que la carafe de l'US 4 (soit une céramique et trois verreries).
La question de savoir si ces objets, dont certains étaient déjà " anciens " au moment du dépôt (cf. infra), ont été placés entiers sur le bûcher peut être posée au vu de la proportion de fragments de pieds ou de fonds par rapport à ceux de lèvres. Le cas du fond en sigillée CE 005 (fig. 14, no 12) est d'ailleurs emblématique, il s'agit probablement d'un élément retaillé pour former une sorte de disque... Une autre particularité, déjà soulignée ailleurs (Tuffreau-Libre, 2001), notamment pour les tombes d'enfants comme c'est le cas ici, est la présence de quatre "vases miniatures " (CE 003, 006, 011 et 015 - fig. 14 , nos $5,1,8$ et 7 ), qui ne forment toutefois pas la totalité du lot et dont aucun fragment ne recolle avec un vase de F1. Cette notion est d'ailleurs complexe à appliquer sur les vases en sigillée (CE 003 et 004 - fig. 14, $\mathrm{n}^{\text {os }} 5$ et 6 ), car ici la petite taille participe à la définition du type dans des "services" (Vernhet, 1976; Bet et Delor, 2000), où sont associées coupelles, coupes et assiettes (la forme Walters 80 étant par exemple la "réduction » ou le «petit module» de la forme Walters 79).

La monnaie s'étant révélée illisible, il appartient au reste du mobilier de fournir des éléments de datation. Lélément a priori le plus récent est le gobelet métallescent CE 009 (fig. 14, no 9; forme Bet 312; Brulet et al., 2010 : p. 312), qui n'est pas produit avant la seconde moitié du $\mathrm{II}^{\mathrm{e}}$ siècle. Cette date relativement tardive est également confirmée par la présence de formes sigillées Drag. 38 et Walters 80 (Bet 31; Bet et Delor, 2000 : p. 480; Brulet et al., 2010 : p. 122) et de probables sigillées des ateliers du sud de la Gaule, dont la production s'est poursuivie largement dans la seconde moitié du II ${ }^{\mathrm{e}}$ siècle $(90$ à 200 pour la forme Ve D2, Genin 2007 : 337; 80-90 à 200 pour la forme Ve C1, Genin, 2007 : 330). Dans ce contexte, la présence d'une céramique à vernis plombifère (Déchelette, 1904 : vases moulés, Planche IV; Brulet et al. 2010 : p. 288-293) et d'un vase en verre bleu foncé, même si certaines identifications restent à confirmer, correspond à la réutilisation d'objets " anciens " conservés depuis une ou deux générations. Les références nous manquent pour les gobelets en céramique fine, dont le médiocre état de conservation et la fragmentation ne favorisent d'ailleurs pas l'identification. Il semble cependant que des parallèles puissent être trouvés sur des sites normands (" gobelets-sacs »: Herment, 1995) ou dans l'atelier d'Agen (Jacques, 2000), dans tous les cas il s'agit de productions $\mathrm{du} \mathrm{II}^{\mathrm{e}}$ siècle, se poursuivant éventuellement au III ${ }^{\mathrm{e}}$. La céramique commune carénée a son pendant identique dans le comblement du puits du site du Barillet à Gétigné (Loire-Atlantique), datable de la première moitié du $\mathrm{II}^{\mathrm{e}}$ siècle (Devals, 2003). Ce type très fréquent est toutefois produit durant une longue période, aux $\mathrm{II}^{\mathrm{e}}$ et $\mathrm{III}^{\mathrm{e}}$ siècles. Pour la figurine, les éléments les plus proches (Herbert, 1995 : $\mathrm{n}^{\circ} 3$; Rouvier-Jeanlin, $1972: \mathrm{n}^{\text {os }} 36$ et 38) sont attribués au 
début du II ${ }^{\mathrm{e}}$ siècle. Concernant les verreries, si les deux urnes appartiennent à des types très courants produits dès la fin du $\mathrm{I}^{\text {er }}$ siècle (Gendron, 1970; Sennequier, 1985), il semble que la carafe VR 006 (fig. 13; forme Isings 88b) corresponde à un type n'apparaissant qu'au II $^{\mathrm{e}}$ siècle, et plus probablement dans la deuxième moitié de ce siècle (Vanpeene, 1993).

\section{DU BÛCHER À LA TOMBE : CARACTÉRISATION DE L'ENSEMBLE FUNÉRAIRE}

En l'absence d'un décapage exhaustif de leurs environs, il reste difficile d'affirmer que ces trois fosses constituent un petit ensemble funéraire isolé, mais le fait ne serait pas exceptionnel. Dans les Pays de la Loire (fig. 17), et grâce à la recherche archéologique menée ces dernières années sur les emprises des grands travaux d'aménagement, le nombre de découvertes de dépôts de crémation isolés a notablement augmenté et confirme les thèses des chercheurs (Galliou, 1989; Monteil, 2004) qui, bien que ne disposant que d'une documentation le plus souvent ancienne et lacunaire, supposaient la coexistence à l'époque romaine des tombes isolées et des nécropoles. Dans la région nantaise, notamment à SaintHerblain (Guérin, 2005), Orvault (Viau, 2005), Carquefou (Le Goff, 2007; Valais, 2008), Nantes (Levillayer, 2012) et au Loroux-Bottereau (Viau, 2012), plusieurs diagnostics et fouilles préventives ont récemment donné lieu à de telles trouvailles. Il s'agit le plus souvent d'urnes en fosse sépulcrale localisées, comme à Gorges, à proximité d'un site antique reconnu, et couramment installées près d'un fossé, comme sur la ZAC de La Haute-Forêt à Carquefou, ou d'un axe de circulation, tel qu'il a pu être constaté sur la ZAC des Garettes à Orvault. Sur le site d'aménagement de la ZAC d'Ar Mor à Saint-Herblain, c'est dans le comblement même d'un fossé qu'une sépulture à crémation a été mise au jour. Le caractère apparemment isolé des sépultures de Gorges doit cependant être considéré avec prudence. Dans la nécropole de la Mothe-Achard, la fouille de 2014 a livré non seulement des ensembles groupés de fosses sépulcrales, mais aussi des tombes implantées à l'écart de ceux-ci, comme souvent en Gaule romaine. Il convient d'ailleurs de souligner que des ossuaires en contenant de verre y étaient enfouis (renseignements : P. Vialet et É. Cabot).

Au Marais, la disposition des fosses alignées sur les bords d'un fossé de parcellaires, à faible distance de celui-ci, suggère une probable installation en pied de talus. Aucun bloc de pierre ou quelconque élément mobilier susceptible de signaler l'emplacement des fosses n'a été découvert lors du décapage. De même, l'extension effectuée autour des structures n'a pas permis d'identifier le lieu de la crémation.
Des indications relatives au bûcher ont cependant été obtenues par l'analyse anthracologique des charbons de bois contenus dans les fosses, qui atteste essentiellement la présence d'essences de bois à combustion lente tels que le chêne ou le hêtre. Quelques fragments d'orme, d'aulne ou de noisetier ont également été mis en évidence. Ces éléments correspondent à ce qui est le plus souvent observé pour les crémations en Gaule, à savoir l'utilisation de bois à combustion lente associé à des essences plus facilement inflammables, permettant d'entretenir ou d'allumer le bûcher (Bel et al., 2009).

Comme l'a démontré la fouille des deux urnes de Gorges, seuls certains fragments d'os appartenant à deux sujets âgés de 3 à 6 ans (du bloc crânio-facial, des os longs) ont été déposés après crémation. Cette sélection apparente des os pourrait témoigner d'un tri volontaire, mais elle peut également résulter d'une plus grande facilité de repérage des fragments d'ossements les plus gros lors de la collecte sur le bûcher (Duday et al., 2000). Le rituel funéraire consistant au prélèvement sélectif des restes osseux est toutefois connu par l'ethnologie (Grévin, 2004). De même, les textes anciens précisent qu'il était courant de trier et de laver les os des défunts avec du lait ou du vin avant de les enfouir (Bel, 2002 : 96). Ce n'est pas le cas par exemple sur la nécropole de La Mothe-Achard en Vendée où, dans leur majorité, les os n'ont pas été séparés des résidus de combustion lors du prélèvement sur le bûcher. Les restes osseux y ont essentiellement été recueillis de manière aléatoire, par poignées, et déposés dans l'urne sans arrangement particulier (Vialet, 2010).

\section{Un dépôt funéraire associé à un dépôt de résidus de combustion ou à une fosse rituelle?}

L'ossuaire en verre de la tombe F3 était fermé par un second vase en verre retourné pour lui servir de couvercle. Les diamètres des deux récipients s'adaptant parfaitement, l'herméticité de l'ensemble était assurée. Ils ont ensuite été disposés dans une céramique modelée utilisée comme réceptacle.

Cette pratique qui consiste à abriter l'urne dans un contenant en terre cuite, en bois ou en pierre est largement attestée dans l'Antiquité. Dans les Pays de la Loire, des vases ossuaires en verre insérés dans des céramiques ont été découverts notamment à Liré dans le Maine-et-Loire (Monteil, 2004 : 117) et à La Mothe-Achard en Vendée (Vialet, 2010). Dans cette dernière nécropole, une urne en verre était installée dans la partie inférieure d'une cruche en céramique et, sur la fouille contiguë réalisée en 2014, un vase en verre était couvert par une coupe en céramique. Une autre urne de la même matière était protégée par un coffrage révélé par la présence de petits clous disposés circulairement autour du 


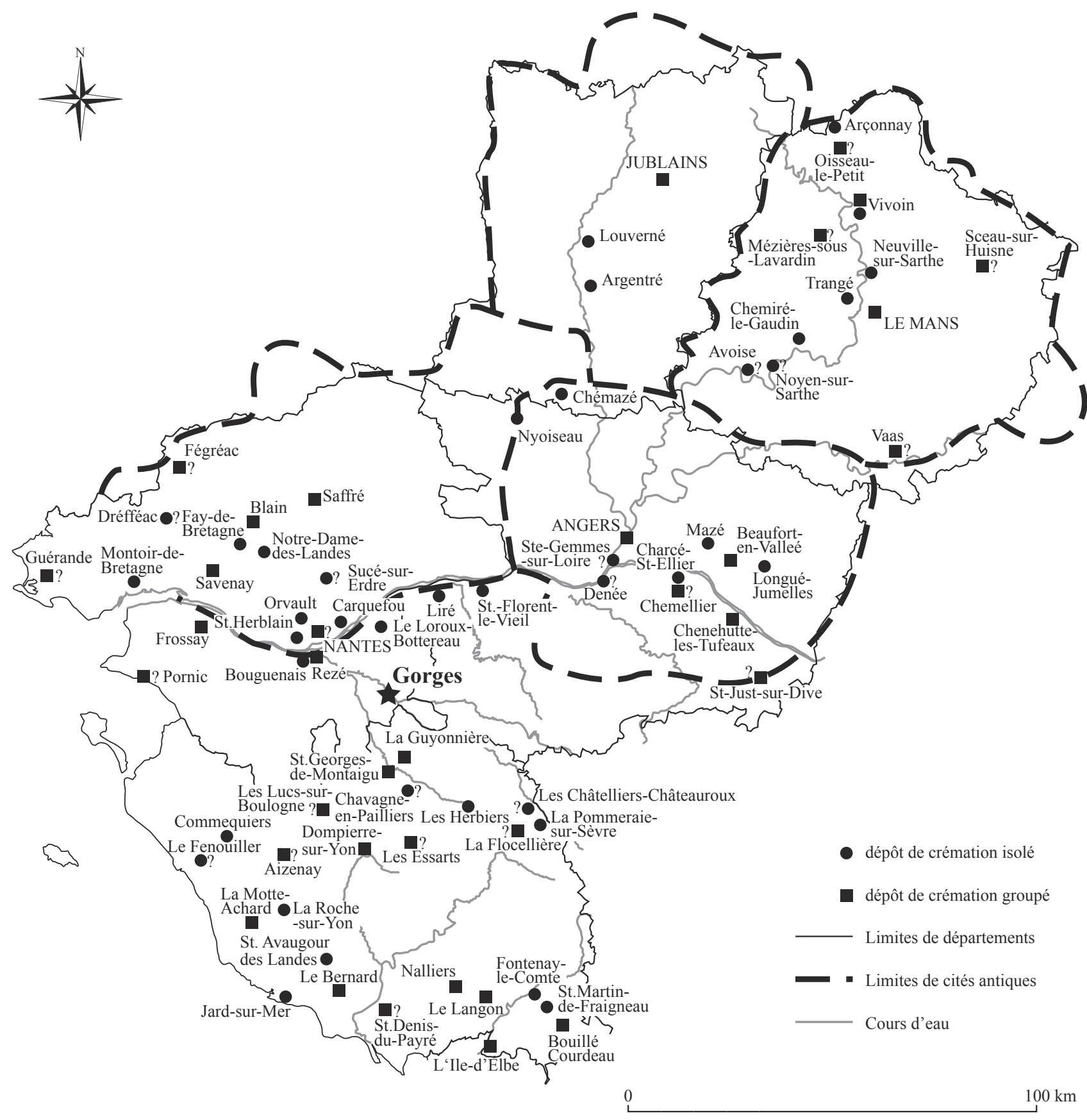

Figure 17 : Gorges, «Le Marais » : cartographie des découvertes de dépôts de crémation gallo-romains isolés et groupés en Pays-de-la-Loire. DAO : J. Cornec, d'après Monteil, 2004.

Figure 17: Cartography of the discoveries of gallo-roman isolated and grouped deposits of cremation.

niveau d'ouverture du récipient (renseignements : P. Vialet et É. Cabot).

Dans la nécropole du Gardou à La Guyonnière (Vendée), deux des trois urnes de verre fouillées comportaient un couvercle constitué par une coupe placée à l'envers et étaient probablement protégées par un coffrage ou du moins un support (Hervé-Monteil, 2011). Sur ce site, deux céramiques parfaitement emboîtées renfermaient également une urne en terre cuite, l'ensemble étant lui-même vraisemblablement protégé par un élément en matière périssable de type panier.

À Chantepie, en Ille-et-Vilaine (Blanchet, 2004), l'espace compris entre un vase ossuaire et les parois internes du 
réceptacle contenait des esquilles osseuses alors qu’à Gorges, seules des cendres y ont été trouvées. Ces cendres, possiblement incandescentes au moment de son dépôt, auraient pu également être à l'origine de la désagrégation du vase ossuaire (fig. 18). Une concentration de morceaux de bois d'aspect charbonneux pourrait en outre indiquer la fermeture du vase réceptacle par une pièce de bois.

Dans la tombe F3 de Gorges, la céramique a ensuite ellemême été placée verticalement au milieu d'un dépôt de résidus de combustion, composé de cendres et de charbons de bois et remplissant la partie inférieure de la fosse. Cette couche ne contenant aucun os, la question de sa provenance se pose, à savoir si ces matériaux ont été extraits du bûcher et dans ce cas auraient fait l'objet d'un tri préalable.

Une cruche en verre, brisée et posée elle aussi verticalement à côté du vase réceptacle, ne présente aucun signe d'un passage sur le bûcher; son anse est absente. La concentration des fragments semble indiquer que la cruche s'est probablement cassée à cause de la pression du comblement, peut-être lors de l'effondrement de l'élément de bois protégeant vraisemblablement la partie médiane de la tombe. Ce mobilier secondaire a pu être déposé après libations lors de l'enfouissement ou pour attester les repas de commémoration à venir.

Pour comparaison, on peut citer l'unguentarium placé contre une urne mise au jour sur la nécropole de la rue des Tuileries à Lyon (Mège et Robin, 2009). L'état de conservation de cette verrerie a permis d'affirmer qu'elle n'avait pas subi de passage au feu et qu'il s'agissait donc d'un objet déposé lors du rituel d'inhumation définitif des restes du défunt. Par ailleurs, deux hypothèses sont envisageables quant à l'absence de l'anse de la cruche : soit la réutilisation d'un récipient abîmé, soit la mutilation volontaire, qui s'avère être une pratique fréquente dans le domaine cultuel et funéraire (Bel, 2002; Blaizot, 2009).

La couche charbonneuse de la partie inférieure de la fosse a livré en outre deux possibles fragments de ferrures pouvant constituer les vestiges d'un coffret de bois brûlé dans le bûcher, mais on ne peut pas non plus exclure qu'il s'agisse d'éléments métalliques fixés sur des pièces de bois de récupération ayant servi de combustible. À moins qu'il ne s'agisse de parties métalliques appartenant à un coffrage de bois renfermant le vase contenant l'ossuaire. En effet, si l'existence d'un tel coffrage n'a pu être mise en évidence lors de la fouille, le sédiment charbonneux compris dans l'espace entre le réceptacle et le fond de fosse tendrait à suggérer la présence d'un fond en bois ou en vannerie comme ce qui a pu être pressenti aux Herbiers (Jégo, 2000 ; 2001), à La Guyonnière (Hervé-Monteil, 2011) et à La Mothe-Achard (Vialet, 2010) en Vendée ou à Vivoin dans la Sarthe (Aubry, 1999).

Enfin, une monnaie usée était posée à plat un peu audessus de la cruche en verre. Cette disposition particulière

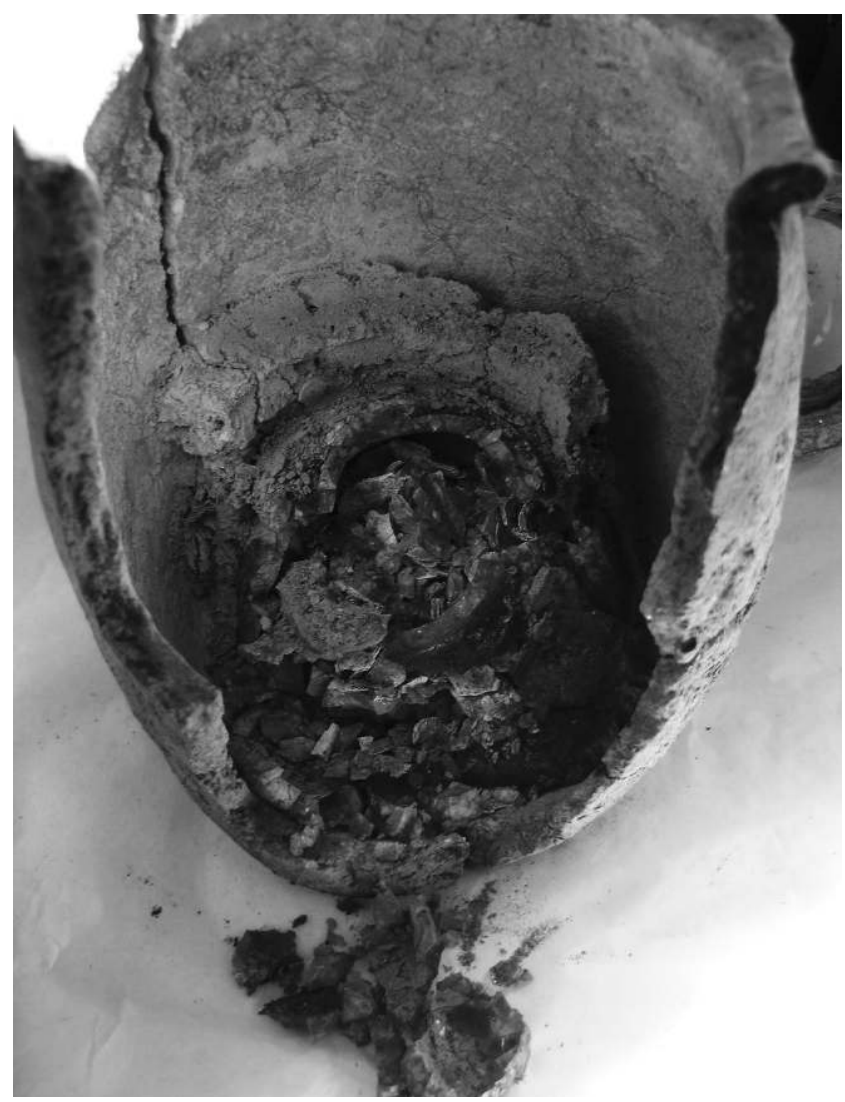

Figure 18 : Gorges, "Le Marais ": désagrégation de la coupe ossuaire de la fosse F3.Cliché : I. Le Goff.

Figure 18: Disintegration of the ossuary cup of the pit F3.

constitue un argument supplémentaire pour suggérer l'existence d'un plancher à l'interface des US 2 et 4. La découverte de monnaies dans les structures de crémation n'est pas fréquente dans les Pays de la Loire. On n'en signale que quelques exemplaires sur les nécropoles de Vivoin (Aubry, 1999), de La Mothe-Achard (Vialet, 2010) ainsi qu’à La Guyonnière (Hervé-Monteil, 2011).

La fine couche d'argile (US 3) provenant de l'encaissant qui sépare les couches charbonneuses inférieure et supérieure indique vraisemblablement la mise en place d'un élément de bois recouvrant le vase réceptacle et l'éventuel coffrage contenant l'ossuaire.

Depuis quelques années, l'observation attentive des remplissages de certaines structures a permis de mettre en évidence l'usage du bois dans les tombes à crémation, cela dès l'époque laténienne, que ce soit sous forme de coffrage ou de simple plancher (Le Goff, 2012). De tels dispositifs en matériaux périssables ou non, dans ce cas constitués par des tegulae, sont par ailleurs connus dans le Sud de la France (Blaizot et al., 2009 : 190).

La partie supérieure de la tombe a livré un dépôt aggloméré d'objets fragmentés et ayant fortement chauffé ou 
fondu. La concentration de cet amas incite à penser qu'il a été placé dans un contenant en matériau périssable de type sac en toile ou en cuir.

De tels dépôts sont parfois introduits dans un vase, mais le site de Feuillis, à Saint-Priest (Rhône), comporte un dépôt en tous points comparables à celui du Marais (Blaizot et al., 2009 : 214). Plus proche, la fouille de la nécropole de La Mothe-Achard a livré récemment un amas similaire dans une fosse à crémation. Le dépôt était par contre excentré par rapport à l'urne en verre (renseignements: P. Vialet et É. Cabot).

Les fragments de céramiques et de verreries identifiés correspondent à des récipients lacunaires de petite taille. La présence de céramiques miniatures ou de petites dimensions est souvent observée, non seulement comme ici dans des sépultures enfantines, mais également dans des tombes d'adultes. On relève notamment un vase miniature déposé en accompagnement dans une des sépultures d'Évreux (Pluton et al., 2008). Si la possibilité qu'il s'agisse d'une sorte de dînette ne peut être écartée, il demeure vraisemblable qu'à l'époque romaine, des récipients de ce type aient été fabriqués dans un but cultuel (Tuffreau-Libre, 2001). On trouve de tels dépôts dans les édifices sacrés et dans les fosses cultuelles associées aux sépultures (Blaizot et al., 2009 : 242-243).

Les verreries fondues sont généralement interprétées comme des fioles de parfums versés sur le bûcher et/ou sur les ossements pendant et à la fin de la crémation, selon un rituel destiné au passage du défunt dans l'au-delà (Scheid, 1984; Galliou, 1989 : 44). Les récipients étaient ensuite eux-mêmes placés sur le bûcher ou dans la sépulture.

Une figurine de Vénus Anadyomène, presque complète mais brûlée et brisée, provient également de l'amas. Plutôt rares, quelques cas de figurines trouvées dans ou près de dépôts de crémation sont cependant mentionnés, notamment à Saint-Marcel dans l'Indre où une tombe était signalée par plusieurs figurines en terre cuite (Allain et al., 1992 : 172-183; Durand, 2013 : 32) ou à Soissons dans l'Aisne (Gissinger, 2012 : 38-39). Dans la nécropole du Clos au Duc à Évreux (Eure), une sépulture a livré une figurine en terre cuite représentant un volatile (Pluton et al., 2008). Une fonction apotropaïque est évoquée pour ce type d'objet, plaçant ainsi le défunt sous la protection de la divinité représentée. Ces figurines sont par ailleurs souvent associées à des tombes d'enfants (Blaizot et al., 2009: 82).

La présence d'une petite perle en verre qui, contrairement au reste, ne semble pas avoir chauffé, mérite d'être relevée car de nombreux cas semblables sont signalés. Des perles en verre ont été identifiées dans la région à La Mothe-Achard en Vendée (Vialet, 2010), à Vivoin dans la Sarthe (Aubry, 1999) et à Liré dans le Maine-et-Loire (Monteil, 2004). On peut évoquer un montage en boucle d'oreille, mais une fonc- tion prophylactique peut aussi être envisagée (Lintz, 2001 : 54).

Le dernier type de mobilier intégré au dépôt consiste en sept petits galets ovoïdes. Bien que peu fréquente, la présence de tels objets est parfois observée dans les tombes à crémation gallo-romaines. Galliou (1989 : 46) relève plusieurs exemples de galets ou d'objets ronds dont la fonction serait de remplacer les monnaies. On peut aussi les interpréter comme de possibles éléments de jeu (Sennequier, 1985), dont l'usage serait comparable à celui des billes de verre, mises au jour notamment à Vivoin (Aubry, 1999), ou de terre cuite (Lintz, 2001).

Outre des esquilles osseuses calcinées malheureusement non identifiables, le comblement cendreux et charbonneux de la fosse F1 a livré du mobilier brûlé pour lequel l'étude a montré l'existence de liaisons par collage avec les céramiques et verreries recueillies dans le dépôt de surface de la tombe F3. On a donc déposé dans cette fosse une partie des éléments qui ont par ailleurs été récoltés de manière sélective et déposés dans la sépulture F3.

$\mathrm{Si}$ les restes osseux correspondent à des os humains, le remplissage de la fosse $\mathrm{F} 1$ constituerait un dépôt de résidus de crémation lié à la sépulture F3.

Des cas similaires ont été identifiés à Pontarion (Creuse), à Sauviat-sur-Vige (Haute-Vienne) et à Concèze (Corrèze) où les résidus de la crémation étaient également placés dans une ou deux fosses voisines de la tombe (Lintz, 2001 : 52). Une association de dépôts de résidus avec les structures de crémation voisines a également pu être établie sur les nécropoles des Landes à La Mothe-Achard en Vendée (Vialet, 2010), de la Butte Saint-Jean à Soissons (Gissinger, 2012) et aux Quaires, dans le Puy-de-Dôme (Blaizot, 2009 : 326-327).

Par contre, s'il s'agit d'ossements animaux, la fosse F1 peut être interprétée comme une fosse rituelle destinée à recueillir les vestiges de la cérémonie des funérailles ou d'une fête commémorative postérieure (Scheid, 2005 : 78). Des exemples similaires de dépôts associant résidus de combustion exempts de restes osseux humains et vaisselle de très petite taille brisée intentionnellement sont signalés dans de nombreux ensembles funéraires (Blaizot, 2009 : 242-243).

La présence de deux grains de blé et d'un pépin de raisin parmi les charbons de bois a été relevée, mais on ne peut déterminer s'il s'agit de dépôts alimentaires ou de matériaux utilisés pour la mise à feu du bûcher.

\section{Un deuxième dépôt secondaire de crémation}

La composition de la tombe F2 est beaucoup plus simple, puisqu'elle ne comporte qu'un petit vase ossuaire, probablement inclus dans un contenant périssable et placé dans une fosse peu profonde. Les ossements déposés dans le récipient 
correspondent à ceux d'un enfant décédé à un âge compris entre 2 et 6 ans.

À côté de l'urne, un amas de clous en fer, visiblement volontairement sélectionnés et regroupés, a été disposé. Cet amas comprenait des clous d'assemblage, quelques clous de semelle et un clou décoratif. Leur présence en elle-même n'est pas surprenante : les plus grands clous peuvent provenir de bois récupérés, utilisés pour la crémation, de panneaux et autres éléments décoratifs ou encore d'étagères installées sur le bûcher. De même, les clous de semelle ont pu appartenir aux chaussures portées par le défunt.

Contrairement à ce qui est le plus fréquent, à savoir des clous mêlés aux résidus de crémation de manière disparate ou des éléments d'assemblage de coffrage, le regroupement particulier au sein de la fosse, observé dans le cas présent, montre une certaine analogie avec des dépôts de clous agencés d'une manière particulière dans certaines sépultures à crémation dont la signification reste discutée (Loridant, 2001).

Dans le dépôt de la fosse F2, la petite urne en céramique contenait des restes de la calotte crânienne d'un enfant d'âge comparable à celui qui était enterré tout à côté. L'étude ostéologique n'a pas permis d'établir avec certitude une corrélation entre les deux ensembles d'ossements. En outre, des différences de coloration semblent désigner deux individus distincts. Différents paramètres nous engagent cependant à relativiser cette interprétation. En effet, la recherche actuelle sur les crématoriums ou les crémations traditionnelles montre qu'un crâne exposé au feu peut soit être entièrement préservé, soit s'effondrer, ou encore éclater si la température atteint rapidement un degré élevé (Grévin, 2004). Dans ce cas, les os peuvent donc être plus ou moins brûlés en fonction de leur localisation et de leur position par rapport au foyer et n'ont donc pas obligatoirement la même couleur à l'issue de la combustion. On ne peut également exclure un brassage des ossements pendant la crémation, impliquant une combustion hétérogène susceptible d'induire des différences de coloration de la représentation osseuse lors du prélèvement des restes du défunt destinés au dépôt secondaire (Blaizot, 2005).

\section{Conclusion}

L'aire sépulcrale du Marais présente des caractéristiques singulières et, pour tout dire, assez inédites pour la connaissance des rites funéraires d'époque romaine dans le nord-ouest de la France, non seulement en raison d'une concentration de matériel inhabituelle pour la région, mais également au regard de son organisation particulière.

Cet ensemble, composé de deux excavations ayant accueilli des dépôts de crémation et d'une fosse contenant du mobilier brûlé dans une couche charbonneuse, reflète un enchaînement de gestes funéraires complexes.

Dans les deux tombes, chaque élément, que ce soit les ossements ou les dépôts de mobilier, a fait l'objet d'un tri préalable à l'enfouissement.

Les recollages inter-structures du mobilier ont permis d'établir que la fosse était liée au moins à l'une des tombes, qui se présente sous une forme particulièrement élaborée rarement reconnue sur les sites ayant livré de telles structures à crémation.

Le mobilier associé à cette tombe, constitué à la fois de dépôts primaires et secondaires rassemble des caractéristiques assez exceptionnelles dans le corpus régional. Le faible pourcentage de dépôts de crémation accompagnés d'objets constaté dans une synthèse ancienne (Galliou, 1989 : 50) est d'ailleurs confirmé par les fouilles et diagnostics archéologiques récents.

Linterprétation du dépôt d'objets brûlés disposé dans la partie supérieure et de la fosse charbonneuse demeure sujette à discussion. Les esquilles osseuses présentes dans cette dernière n'ayant pu être identifiées, il peut s'agir de dépôts de résidus de crémation ou de commémoration postérieure aux funérailles.

\section{Bibliographie}

Allain J., Fauduet I. et Truffeau-Libre M., 1992 - « La nécropole gallo-romaine du Champ de l'Image à Argentomagus (Saint-Marcel, Indre) ", Mémoire 1 du musée d'Argentomagus, $3^{\mathrm{e}}$ supplément à la Revue archéologique du centre de la France, Saint-Marcel, 237 p.

Aubry B., 1999 - La Petite Nèmerie à Vivoin, (Sarthe), Rapport de fouille préventive, Nantes, Afan/SRA Pays-de-la-Loire.

Bel V., 2002 - «Pratiques funéraires du Haut-Empire dans le Midi de la Gaule : la nécropole gallo-romaine du Valladas à Saint-Paul-Trois-Chateaux (Drôme) ", Monographies d'archéologie méditerranéenne, 11, Lattes, ARALO, 539 p.

Bel V., Blaizot F., Bonnet C., Ganol M.-E., Georges P., Gisclon J.-L., Lisfranc R., Richier A. et Wittman A., 2009 - "L'étape de la crémation : les bûchers funéraires ", in Blaizot F. (dir.), Pratiques et espaces funéraires dans le centre et le sud-est de la Gaule durant l'Antiquité, Paris, éditions du CNRS, p. 89-150 (Gallia, 66.1).

Bellanger P., 2002 - Déviation nord et nord-est de Clisson (LoireAtlantique), Rapport de diagnostic archéologique, Nantes, Inrap/ SRA Pays de la Loire.

Bet P. et Delor A., 2000 - « La typologie de la sigillée lisse de Lezoux et de la Gaule centrale du Haut-Empire, révision décennale ", SFECAG, Actes du Congrès de Libourne, p. 461484. 
Blaizot F. et Tranoy L., 2004 - « La notion de sépultures au Haut-Empire : identification et interprétation des structures funéraires liées à la crémation ", in BARAY L. (dir.), Archéologie des pratiques funéraires : approches critiques, Actes de la table ronde, Glux-en-Glenne, 7-9 juin 2001 Glux-en-Glenne, p. 171-187 (Bibracte, 9).

Blaizot F., 2005 - "Contribution à la connaissance des modes de dislocation et de destruction du squelette pendant la crémation : l'apport du bûcher funéraire en fosse du Néolithique final à Reichstett-Mundolsheim (Bas-Rhin) ", Bulletins et Mémoires de la Société d'anthropologie de Paris, 17, fasc. 1- 2, Paris, Société d'anthropologie de Paris, Musée de l'Homme, p. 13-35.

Blaizot F., 2009 - « L'image sociale et culturelle des pratiques funéraires : expressions et évolutions de la société romaine dans le sud-est de la Gaule ", in Blaizot F. (dir.), Pratiques et espaces funéraires dans le centre et le sud-est de la Gaule durant l'Antiquité, Paris, éditions du CNRS, p. 311-344 (Gallia, 66.1).

Blaizot F., Bel V., Bonnet C., Wittman A., Georges P., Gisclon J.-L., Tranoy L. et Vieugue J., 2009 - « Structures secondaires et structures accessoires ", in Blaizot F. (dir.), Pratiques et espaces funéraires dans le centre et le sud-est de la Gaule durant l'Antiquité, Paris, éditions du CNRS, p. 175-252 (Gallia, 66.1).

Blanchet S., 2004 - Les rives du Blosne à Chantepie (Ille-etVilaine), Rapport de fouille préventive, Nantes, Inrap/SRA Bretagne.

Brulet R., Vilvorder F. et Delage R., 2010 - La céramique romaine en Gaule du Nord, la vaisselle à large diffusion, Thurnout (Belgique), Brepols publisher, $462 \mathrm{p}$.

Déchelette J., 1904 - Vases céramiques ornés de la Gaule romaine, 5 fasc., Paris (rééd. 1979), 2 vol.

Depierre G., 2014 - Crémation et archéologie; Nouvelles alternatives méthodologiques en ostéologie humaines, Éditions Universitaires de Dijon, Collection Art, Archéologie et Patrimoine, Dijon, 654 p.

Devals C., 2003 - L'enclos gallo-romain du Barillet à Gétigné, Rapport de fouille préventive, Nantes, Inrap/SRA Pays de la Loire.

Duday H., Depierre G. et Janin T., 2000 - Validation des paramètres de quantification, protocoles et stratégies dans l'étude anthropologique des sépultures secondaires à incinération. L'exemple des nécropoles protohistoriques du Midi de la France, in Dedet B., Gruat P.-H., Marchand G., Py M. et Schwaller M. (dir.), Archéologie de la mort, Archéologie de la tombe au premier Age du Fer, Actes du XXI colloque de l'AFEAF, Conques-Montrozier, 1997, Monographies d'Archéologie Méditerranéenne, 5, Lattes, p. 7-29.

Durand R., 2013 - "La nécropole gallo-romaine du Champ de l'Image et les pratiques funéraires à Argentomagus (SaintMarcel, Indre) : données anciennes et nouvelles approches ", in FERDiÈre A. (dir.), Ensembles funéraires gallo-romains de la Loire-Moyenne-II-, 44e supplément à la Revue archéologique du centre de la France, p. 25-50.

Galliou P., 1989 - «Les tombes romaines d'Armorique, Essai de sociologie et d'économie de la mort ", Documents d'archéologie française, $\mathrm{n}^{\circ} 17$, Paris, $204 \mathrm{p}$.

Gejvall N.-G., 1963 - "Cremations », in Brothwell D., Higgs E., Science in archeology. A comprehensive survey of progress and research, p. 379-390.

Gendron C., 1970 - La verrerie gallo-romaine sur le Golfe des Pictons, Mémoire de maîtrise d'histoire de l'art et d'archéologie, université de Poitiers.

Genin M. (éd.), 2007 - La Graufesenque (Millau, Aveyron) : vol. II sigillées lisses et autres productions, Bordeaux, Fédération Aquitania, $589 \mathrm{p}$.

Gissinger B. (dir.), 2012 - «Fouille d'un quartier funéraire des I $^{\text {er }}$ et II $^{\text {e }}$ siècles dans le suburbium de Soissons/Augusta Suessionum : aires de crémation et inhumations d'enfants ", in Nécropoles et sociétés : cinq ensembles funéraires des provinces de Gaule (ier-ve siècle apr. J.-C), Gallia, 69.1, Paris, éditions du CNRS, p. 3-68.

GrÉvin G., 2005 - «Les crémations sur bûcher dans l'Antiquité à la lumière de l'ethnoarchéologie ", in BACHelot L., Le GofF I. et Tenu A. (éd.), Entre mondes orientaux et classiques : la place de la crémation, Actes du colloque international de Nanterre, Strasbourg, Université Marc-Bloch, coll. « Ktema, 30 ", p. 15-20.

Guérin F., 2005 - Aménagement du site d'Ar Mor à Saint-Herblain (Loire-Atlantique), Rapport de diagnostic archéologique, Nantes, Inrap/ SRA Pays de la Loire.

Guitton D., Thébaud S., Landreau G., Véquaud B. et coll., 2015 - «Permanence de la céramique non tournée en territoire picton du Haut-Empire à la fin du haut Moyen Âge : un phénomène d'ampleur lié à un terroir ", in Joly M. et SEGUIER J.-M. (dir.), Les céramiques non tournées en Gaule romaine dans leur contexte social, économique et culturel : entre tradition et innovation, Actes du colloque tenu les 25 et 26 novembre 2010 à Paris, Tours, 55² supplément à la Revue archéologique du centre de la France, p. 85-124.

Herbert N., 1995 - Les figurines en terre cuite d'époque romaine du musée Dobrée, Mémoire de maîtrise, Nantes, vol. 1 texte, 212 p., vol. 2 planches, 82 pl.

Herment H., 1995 - «Aperçu de la céramique ébroïcienne à travers le site du square Georges-Brassens « la Médiathèque " à Évreux (Eure, Haute-Normandie) ", SFECAG, Actes du Congrès de Rouen, p. 119-128.

Hervé-Monteil M.-L., 2011 - Le Gardou à La Guyonnière (Vendée), Rapport de fouille préventive, Nantes, Inrap/SRA Pays de la Loire. 
JACQues P., 2000 - «Une officine de potiers à Agen (Lot-etGaronne) au III $^{\mathrm{e}}$ siècle?", SFECAG, Actes du congrès de Libourne, p. 53-73.

JÉgo L., 2000 - Bel-Air 2 aux Herbiers (Vendée), Autoroute A.87, Tronçon 2, Saint-Laurent-sur-Sèvre, La Roche-sur-Yon, Rapport de diagnostic archéologique, Nantes, Inrap/SRA Pays de la Loire.

Jégo L., 2001 - Bel-Air 2 aux Herbiers (Vendée), Autoroute A.87, Tronçon 2, Saint-Laurent-sur-Sèvre, La Roche-sur-Yon, Rapport de complément de diagnostic archéologique, Nantes, Inrap/ SRA Pays de la Loire.

Le Goff E., 2007 - ZAC de la Haute-Forêt à Carquefou (LoireAtlantique), Rapport de diagnostic archéologique, Nantes, Inrap/ SRA Pays de la Loire.

Le Goff I., 2012 - «Le bois dans les tombes à crémation, approche taphonomique ", in CArré F., Henrion F. (dir.), Le bois dans l'architecture et l'aménagement de la tombe : quelles approches?, Actes de la table ronde à Auxerre, 15-17 octobre 2009, Mémoires publiées par l'AFAM, tome XXIII, p. 241 248.

Levillayer A., 2012 - ZAC Erdre-Porterie à Nantes (LoireAtlantique), Rapport de fouille préventive, Nantes, Archéoloire/ SRA Pays de la Loire.

Lintz G., 2001 - La nécropole gallo-romaine des Sagnes à Pontarion (Creuse), Mémoire XX, 376 p.

LORIDANT F., 2001 - "Autopsie d'une sépulture à incinération : la tombe 11 de la nécropole gallo-romaine de la "Fache des Prés Aulnoys " à Bavay ", in Geoffroy J.-F. et Barbe H. (éd.), Les nécropoles à incinérations en Gaule Belgique, synthèses régionales et méthodologie, Actes du XIX ${ }^{\mathrm{e}}$ colloque international du CRAU tenu les 13 et 14 décembre 1996. Lille, Revue du Nord, hors-série, $\mathrm{n}^{\circ}$ 8, p. 189-196.

Mège C. et Robin L., 2009 - "Un unguentarium-chandelier à fond marqué du $\mathrm{II}^{\mathrm{e}}$ siècle apr. J.-C. découvert à Lyon ", Revue archéologique de l'Est, t. 58, p. 453-459.

Monteil M., 2004 - "Tombes et nécropoles rurales du HautEmpire. Un état des recherches en Pays de la Loire ", Bulletin de la Société archéologique et historique de Nantes et de LoireAtlantique, 139, p. 93-139.

Moorrees C. F. A., Fanning E. A. and Hunt E. E., 1963 "Formation and resorption of three deciduous teeth in children ", American Journal of Physical Anthropology, 21, p. 205-213.

Pluton S., Adrian Y.-M., Kliesch F. et Cottard A., 2008 - « La nécropole gallo-romaine du "Clos au Duc" à Évreux (Eure) : des sépultures du I $^{\mathrm{er}}$ siècle apr. J.-C. ", Revue archéologique de l'Ouest, 25, p. 209-260.

Rouvier-JEANlin M., 1972 - Les figurines gallo-romaines en terre cuite au Musée des Antiquités Nationales, XXIV supplément à Gallia, éditions du CNRS, Paris, 428 p.

Scheid J., 1984 - "Contraria facere : renversements et déplacements dans les rites funéraires ", Annali dell'Istituto Orientale di Napoli, 6, p. 117-139.

Sennequier G., 1985 - Verrerie d'époque romaine, Rouen, Collection des musées départementaux de Seine-Maritime, $211 \mathrm{p}$.

Thébaud S., coll. Simon L., 2012 - « Le mobilier de deux ensembles funéraires en Vendée : les sites du Gardou à La Guyonnière et des Landes à La Mothe-Achard ", SFECAG, Actes du Congrès de Poitiers, Marseille, 2012, p. 243-262.

Tuffreau-Libre M., 2001 - "Les assemblages céramiques dans les nécropoles gallo-romaines ", in Geoffroy J.-F. et BARBÉ H. (éd.), Les nécropoles à incinérations en Gaule Belgique, synthèses régionales et méthodologie, Actes du XIX colloque international du CRAU tenu les 13 et 14 décembre 1996, Lille, Revue du Nord, hors-série n ${ }^{\circ} 8$, p. 179-187.

Valais A., 2008 - La Haute-Forêt à Carquefou (Loire-Atlantique), Rapport de fouille préventive, Nantes, Inrap/ SRA Pays de la Loire.

VANPEENE N., 1993 - Verrerie de la nécropole d'Épiais-Rhus (Vald'Oise). Centre de recherche archéologique du Vexin français, Cahier archéologique n 8 , Guiry-en-Vexin, 101 p.

Vernhet A., 1976 - "Création flavienne de six services à La Graufesenque », Figlina, 1, p. 13-27.

Vialet P., 2010 - L'ensemble funéraire du Haut-Empire des Landes à La Mothe-Achard, La Chapelle-Achard (Vendée), Rapport de fouille préventive, Nantes, Inrap/SRA Pays de la Loire.

VIAu Y., 2005 - ZAC des Garettes à Orvault (Loire-Atlantique), Rapport de diagnostic archéologique, Nantes, Inrap/SRA Pays de la Loire.

VIAU Y., 2012 - ZAC du Plessis au Loroux-Bottereau (LoireAtlantique), Rapport de diagnostic archéologique, Nantes, Inrap/SRA Pays de la Loire.

WAHL J., 1988 - Süderbrarup. Ein Gräberfeld der römischen Kaiserzeit und Völkerwanderungszeit in Angeln, Neumünster, Karl Wachholtz Verlag, 139 p. 
Zusammenfassung: Eine kleine Gruppe römischer Brandgräber der frühen römischen Kaiserzeit bei Gorges „Le Marais“(Loire-Atlantique, Frankreich) - In der Nähe einer ländlichen römischen Siedlung wurden bei Gorges (Loire-Atlantique, Frankreich) in der Flurbezeichnung "Le Marais“ zwei Brandgräber des 2. Jh. n. Chr. entdeckt, die als sekundäre Aschenschüttungen anzusprechen sind. Die eine dieser Brandbestattungen ist als eine gemischte Brandschüttung zu interpretieren, zu der sich eine dritte Grube gesellte, die ebenfalls Überreste derselben Brandbestattung aufwies. Zweifel bestehen hinsichtlich der zweiten Brandbestattung, die ebenso wie die erste Überreste eines Kleinkindes enthielt. Es war nicht möglich zu entscheiden, ob dasselbe Individuum in zwei Gruben in Form von Brandschüttungen beigesetzt wurde oder ob es sich um Brandgräber handelt, in denen zwei verschiedene Individuen beigesetzt worden sind.

Schlüsselwörter: Loire-Atlantique, Frühe und Hohe Kaiserzeit, sekundäre Brandbestattung, Brandschüttung, Aschengrube. 